\title{
Following nuclei through nucleosynthesis: a novel tracing technique
}

\author{
T.M. Sprouse* ${ }^{*}, 2$ M.R. Mumpower, ${ }^{2,3}$ and R. Surman ${ }^{1}$ \\ ${ }^{1}$ Department of Physics, University of Notre Dame, Notre Dame, IN 46556, USA \\ ${ }^{2}$ Theoretical Division, Los Alamos National Laboratory, Los Alamos, NM 87545, USA \\ ${ }^{3}$ Center for Theoretical Astrophysics, Los Alamos National Laboratory, Los Alamos, NM 87545, USA
}

(Dated: August 17, 2020)

\begin{abstract}
Astrophysical nucleosynthesis is a family of diverse processes by which atomic nuclei undergo nuclear reactions and decays to form new nuclei. The complex nature of nucleosynthesis, which can involve as many as tens of thousands of interactions between thousands of nuclei, makes it difficult to study any one of these interactions in isolation using standard approaches. In this work, we present a new technique, nucleosynthesis tracing, that we use to quantify the specific role of individual nuclear reaction, decay, and fission processes in relationship to nucleosynthesis as a whole. We apply this technique to study fission and $\beta^{-}$-decay as they occur in the rapid neutron capture $(r)$ process of nucleosynthesis.
\end{abstract}

\section{INTRODUCTION}

The extreme conditions that can arise in astrophysical environments enable nuclear transmutation processes to take place, by which atomic nuclei interact with their environment or decay to form new nuclei. Insofar as different astrophysical environments may foster certain transmutation processes but not others, these environments may be categorized by the different types of nucleosynthesis that occur in each; one of the primary goals of nuclear astrophysics, then, is to explain how these different nucleosynthesis sources produce all of the chemical elements observed in the universe, beginning with the primordial hydrogen and helium produced during the Big Bang [1].

In the most complex cases, nucleosynthesis can involve many thousands of nuclear species connected by upward of $\sim 100,000$ nuclear transmutation processes by which their abundances may evolve in time. Because the rates at which the different processes occur may depend on the temperature and density of the environment in which the nuclei are situated, as well as the abundances of the different nuclei themselves, nucleosynthesis is an extremely dynamical and nonlinear problem. Nevertheless, through the use of nuclear reaction networks, it is possible to effectively model nucleosynthesis numerically.

More difficult, however, is the problem of isolating and quantifying the role of individual nuclear properties as they influence nucleosynthesis as a whole. This can be especially important because nucleosynthesis is inherently determined by the properties of the nuclei that participate in it, making these properties the focus of a significant number of experimental and theoretical campaigns in nuclear physics (see, e.g., [2 [6] and references therein). By identifying the most crucial nuclear properties for each nucleosynthesis process, these campaigns may be

${ }^{*}$ Los Alamos Center for Space and Earth Science Student Fellow more precisely focused in the near-future on those properties which will most significantly constrain nucleosynthesis simulation predictions.

Past approaches to accomplish this goal have either (1) systematically varied individual or collections of nuclear properties and examined the relative changes to nucleosynthesis, for example in [7-14] or (2) analyzed the overall rate at which different processes (reactions, decays, or fission) occur during nucleosynthesis, such as in [15 17. However, it has not been possible using current techniques to precisely quantify which nuclei, and in what amounts, are affected by individual nuclear properties. Approach (1) inherently modifies the nucleosynthesis simulation itself, insofar as decay and reaction rates themselves are modified. While approach (2) does not affect nucleosynthesis simulations in the same way, it provides only limited insight into which aspects of a simulated abundance pattern are affected.

In this work, we introduce a new framework for nucleosynthesis modeling, nucleosynthesis tracing, that can be applied to supplement these two approaches. Nucleosynthesis tracing enables the robust quantification of which nuclei have participated in an arbitrary collection of nuclear reactions, decays, and/or fission at some point during nucleosynthesis. In Sec. [II] we identify the underlying assumptions of the nucleosynthesis tracing framework and derive the differential equations that define the technique. In Sec. III, we briefly summarize how we have implemented nucleosynthesis tracing as PRISM ${ }^{\text {tr }}$, a modified version of the nuclear reaction network code Portable Routines for Integrated nucleoSynthesis Modeling (PRISM). Finally, we demonstrate several possible applications of nucleosynthesis tracing to rapid neutron capture ( $r$ process) nucleosynthesis in Sec. IV.

\section{THEORY}

Traditional nuclear reaction networks time-evolve the nuclear abundances of a system actively undergoing nu- 
cleosynthesis. These calculations require a number of different input parameters, including an initial composition of nuclei and any relevant nuclear properties, such as nuclear reaction and decay rates. Because these rates may depend on the thermodynamics of the system, it is also necessary to specify and evolve the temperature and density during nucleosynthesis. Reaction networks may also incorporate other environmental properties, such as external heating rates and (anti)neutrino fluxes, into their calculations.

The generalized problem of simulating nucleosynthesis may then be phrased as follows. We assume that each nuclide in a system can be uniquely identified by its proton number, $Z$, and mass number, $A$. To each combination of these $(Z, A)$, we assign an integer, $i$, that indexes the species. The number density of species $i$ at any given time is given as $n_{i}$, and we define the relative abundance of this species as $Y_{i}=n_{i} / \rho N_{A}$, where $\rho$ is the baryon density, and the mass fractions $X_{i}=Y_{i} A_{i}$ sum to 1 , $\sum_{i} X_{i}=1$.

Changes in abundances are enacted by a collection of nuclear transmutation processes, $P$. For each process $p$ in $P$, we require the associated quantities listed below.

1. A rate function $\Lambda_{p}$, which is allowed to depend explicitly on any environmental quantity available to the network, such as temperature, density, or neutrino flux, as appropriate to the process. Insofar as each of these quantities is available to the network as a function of time, the function $\Lambda_{p}$ is implicitly a function of time.

2. A set of nuclear indices, $\mathcal{R}_{p}$, that correspond to the nuclei consumed by the process.

3. A set of nuclear indices, $\mathcal{P}_{p}$, that corresponds to the nuclei produced by the process.

4. A function, $\alpha_{p}(i)$, that gives the number of species with index $i \in \mathcal{R}_{p}$ consumed by the process.

5. A function, $\beta_{p}(i)$, that gives the average number of species with index $i \in \mathcal{P}_{p}$ produced by the process.

Once a specific collection of relevant processes, $P$, is specified, nucleosynthesis is reduced to solving an initial value problem (IVP) for the abundances as a function of time, $Y_{i}(t)$. The abundances at time $t_{0}$ are taken as the initial condition. The set of processes, $P$, completely defines the system of differential equations for the IVP. For each species in the network, $i$, the differential equation defining its evolution in time is given as

$$
\begin{aligned}
\frac{d Y_{i}}{d t}= & -\sum_{\left\{p \in P \mid i \in \mathcal{R}_{p}\right\}}\left(\alpha_{p}(i) \Lambda_{p}(t) \prod_{j \in \mathcal{R}_{p}} Y_{j}(t)^{\alpha_{p}(j)}\right) \\
& +\sum_{\left\{p \in P \mid i \in \mathcal{P}_{p}\right\}}\left(\beta_{p}(i) \Lambda_{p}(t) \prod_{j \in \mathcal{R}_{p}} Y_{j}(t)^{\alpha_{p}(j)}\right),
\end{aligned}
$$

where the first summation is taken over processes in which nuclide $i$ is given in $\mathcal{R}$ and the second over processes in which nuclide $i$ is given in $\mathcal{P}$. Because our notation differs significantly from more commonly adopted forms, we refer to the Appendix for a description of the relationship between Eq. 1 and that used, e.g., in [18].

The specific approaches that are taken to solve this IVP vary across reaction networks, and the ideal numerical methods can be application-dependent. For simulating $r$-process nucleosynthesis, a common approach is to solve an implicit Euler equation using the Newton-Raphson method [18 22], although alternative approaches have also demonstrated success [23 28].

\section{A. Nucleosynthesis Tracing}

In this work, we develop an extension to reaction networks as previously defined. We refer to this extension as a tracing reaction network. This extension, which is laid out below, enables the robust quantification of which nuclei in a system have a nucleosynthetic history involving a particular process or processes, among other possible applications.

We begin by constructing a parallel set of abundances to evolve, denoted as $Y_{\text {traced, } i}$ and referred to as the traced abundances. Physically, the traced abundances identify the subset of the total abundances which have assumed some property, a trace-in condition, during nucleosynthesis. Once a nucleus satisfies the trace-in condition, we add it to the traced abundances. We may also wish to remove nuclei from consideration in the traced abundances after they assume some other property, a trace-out condition. We otherwise evolve the overall abundances according to Eq. 1 and the traced abundances according to a slightly modified form of the same equation. It is also helpful to define the untraced abundances as $Y_{\text {untraced }, i}=Y_{i}-Y_{\text {traced }, i}$.

For the present work, we strictly consider trace-in and trace-out conditions to be the participation in a collection of processes, $P_{\text {in }}$ and $P_{\text {out }}$, respectively. Effectively, we begin tracing a nucleus once it is produced by a process identified in $P_{\text {in }}$, and we continue to follow it throughout all subsequent nucleosynthesis in which it participates. However, if it is consumed by one of the processes in $P_{\text {out }}$, we remove it from the traced abundances, and we do not consider any further nucleosynthesis in which it participates. We also define an additional set of processes, $P_{\text {other }}$, which we define as the set of those processes in $P$ belonging to neither $P_{\text {in }}$ nor $P_{\text {out }}$.

We illustrate schematically how one- and two-body processes in each of $P_{\text {in }}, P_{\text {out }}$, and $P_{\text {other }}$ affect the evolution of the traced abundances in Fig. 1. Processes in $P_{\text {in }}$ and $P_{\text {out }}$ are relatively straightforward, as products are always mapped into the traced and untraced populations, respectively. For one-body processes in $P_{\text {other }}$, the mapping is straightforward as well, as products of traced nuclei are mapped into the traced population, and prod- 


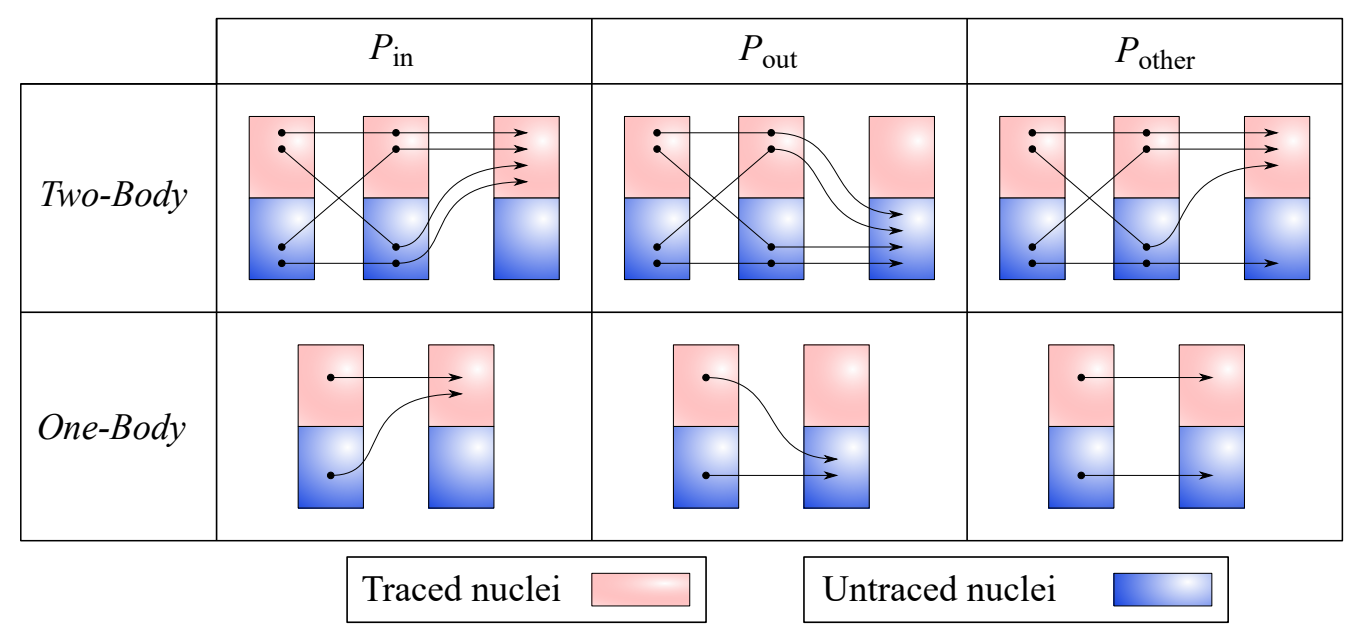

FIG. 1: A schematic diagram of nucleosynthesis tracing. Populations of traced nuclei (top red boxes) and untraced nuclei (bottom blue boxes) interact via one-body and two-body trace-in processes $\left(P_{\text {in }}\right)$, trace-out processes $\left(P_{\text {out }}\right)$, and other processes $\left(P_{\text {other }}\right)$. The filled circles represent nuclei consumed by a process, and the arrowheads represent nuclei produced by a process. For the trace-in processes, products are mapped exclusively to the traced population, while the trace-out processes map products exclusively to the untraced population. All other processes are allowed to map products to either the traced or untraced populations, depending on the populations to which the reactants belong.

ucts of untraced nuclei are mapped into the untraced population.

For two-or-more-body processes, the situation is more complicated. If a traced nucleus interacts with a traced nucleus, then the products clearly should be mapped into the traced population. Likewise, if only untraced nuclei undergo the process, then the products unambiguously belong in the untraced population. It is also possible that some number of untraced nuclei interact with some other traced nuclei, and we are forced to choose what fraction of the products belong to the traced and untraced populations. The simplest choice is to assert that if any nucleus consumed by the process is traced, then the products are always mapped into the traced population, as can be seen in the top-right panel of Fig. 1. This is the choice we explore in the present work, although other meaningful choices are possible.

The system of differential equations describing the evolution of the traced abundances may now be defined under these assumptions. There are four distinct varieties of terms that present themselves in these differential equations, and we construct each of them in turn.

A traced nucleus, $i$, is produced by a process, $p$, in $P_{i n}$. Because we wish to add all of the nuclei produced via this process into the traced network, the relevant term should be identical to that of the total abundance $Y_{i}$, namely

$$
R_{1}=\beta_{p}(i) \Lambda_{p}(t) \prod_{j \in \mathcal{R}_{p}} Y_{j}(t)^{\alpha_{p}(j)}
$$

A traced nucleus, $i$, is produced by a process, $p$, in $P_{\text {out }}$. Because none of the nuclei produced via this process should be mapped into the traced network, this term is simply 0 .
A traced nucleus, $i$, is produced by a process, $p$, in $P_{\text {other }}$. All nuclei produced by this process should be mapped into the traced network unless all of the nuclei involved are untraced. The rate at which only untraced nuclei undergo the process is given by

$$
R_{2}^{\prime}=\beta_{p}(i) \Lambda_{p}(t) \prod_{j \in \mathcal{R}_{p}} Y_{\text {untraced }, j}(t)^{\alpha_{p}(j)} .
$$

The rate at which traced nuclei are produced is the difference between Eq. 22 and Eq. 3.

$$
\begin{aligned}
R_{2}= & \beta_{p}(i) \Lambda_{p}(t) \prod_{j \in \mathcal{R}_{p}} Y_{j}(t)^{\alpha_{p}(j)} \\
& -\beta_{p}(i) \Lambda_{p}(t) \prod_{j \in \mathcal{R}_{p}} Y_{\text {untraced }, j}(t)^{\alpha_{p}(j)} .
\end{aligned}
$$

Expressed in terms of only traced abundances $Y_{\text {traced }, i}$ and overall abundances $Y_{i}$, this reduces to

$$
\begin{aligned}
R_{2}= & \beta_{p}(i) \Lambda_{p}(t) \prod_{j \in \mathcal{R}_{p}} Y_{j}(t)^{\alpha_{p}(j)} \\
& -\beta_{p}(i) \Lambda_{p}(t) \prod_{j \in \mathcal{R}_{p}}\left[Y_{j}(t)-Y_{\text {traced }, j}(t)\right]^{\alpha_{p}(j)} .
\end{aligned}
$$

A traced nucleus, $i$, is consumed by a process, $p$, in $P_{i n}$, $P_{\text {out }}$, or $P_{\text {other }}$. A traced nucleus may interact with any other nucleus, traced or otherwise, to undergo a particular process. As such, the rate at which a traced nucleus is consumed by the process will be in proportion to the overall rate in the ratio $Y_{\text {traced, } i} / Y_{i}$. The term is given by

$$
R_{3}=-\frac{Y_{\text {traced }, i}}{Y_{i}}\left(\alpha_{p}(i) \Lambda_{p}(t) \prod_{j \in \mathcal{R}_{p}} Y_{j}(t)^{\alpha_{p}(j)}\right) .
$$


Because an abundance $Y_{i}$ may be 0 , we rearrange this slightly as

$$
R_{3}=-\alpha_{p}(i) \Lambda_{p}(t) Y_{\text {traced }, i} Y_{i}^{\alpha_{p}(i)-1} \prod_{j \neq i \in \mathcal{R}_{p}} Y_{j}(t)^{\alpha_{p}(j)} .
$$

The system of equations that govern the traced reaction network is a linear sum of all appropriate terms of the forms Eq. 2, Eq. 5, and Eq. 6, together with the system of equations defined in Eq. 1. It is given by

$$
\begin{aligned}
\frac{d Y_{i}}{d t}= & -\sum_{\left\{p \in P \mid i \in \mathcal{R}_{p}\right\}}\left(\alpha_{p}(i) \Lambda_{p}(t) \prod_{j \in \mathcal{R}_{p}} Y_{j}(t)^{\alpha_{p}(j)}\right) \\
& +\sum_{\left\{p \in P \mid i \in \mathcal{P}_{p}\right\}}\left(\beta_{p}(i) \Lambda_{p}(t) \prod_{j \in \mathcal{R}_{p}} Y_{j}(t)^{\alpha_{p}(j)}\right) \\
\frac{d Y_{\text {traced }, i}}{d t}= & -\sum_{\left\{p \in P \mid i \in \mathcal{R}_{p}\right\}}\left[\alpha_{p}(i) \Lambda_{p}(t) Y_{\text {traced }, i} Y_{i}^{\alpha_{p}(i)-1}\right. \\
& +\sum_{\left\{p \in P_{\text {in }} \mid i \in \mathcal{P}_{p}\right\}}\left[\beta_{p \neq i \in \mathcal{R}_{p}} Y_{j}(t)^{\alpha_{p}(j)}\right] \\
& +\sum_{\left\{p \in P_{\text {other }} \mid i \in \mathcal{P}_{p}\right\}}\left[\beta_{p}(i) \Lambda_{p}(t) \prod_{j \in \mathcal{R}_{p}} Y_{j}(t)^{\alpha_{p}(j)} Y_{j}(t)^{\alpha_{p}(j)}\right. \\
& \left.-\beta_{p}(i) \Lambda_{p}(t) \prod_{j \in \mathcal{R}_{p}}\left[Y_{j}(t)-Y_{\text {traced }, j}(t)\right]^{\alpha_{p}(j)}\right]
\end{aligned}
$$

This extended system of equations can then be solved using the same numerical techniques as for traditional network equations (Eq. 1). Both the total and traced abundances evolve mostly separate from each other, with connections between the two mediated by the $P_{\text {in }}$ and $P_{\text {out }}$ processes. Note that because the equations for $\frac{d Y_{i}}{d t}$ are the same as in Eq. 1. they are not affected in any way by the extended network, and simulating the total abundances will not be affected by using the extended network equations. However, the total abundances do arise in the equations for the $\frac{d Y_{\text {traced }, i}}{d t}$, and through this dependence the dynamic interactions of the traced abundances with the total abundances are effectively captured.

\section{NUCLEOSYNTHESIS AND PRISM}

For the demonstrated applications of the nucleosynthesis tracing framework presented in Sec. [V] we use an updated version of the reaction network code PRISM 16. 29, 30 denoted as PRISM ${ }^{\text {tr }}$. The extended network equations defining the evolution of the traced abundances, summarized as Eq. 8, are structurally very similar to those of the total abundances, Eq. 77. Numerically, we solve both the total abundances and the traced abundances over a series of discrete timesteps by solving an implicit Euler equation using the Newton-Raphson method. In addition to solving for the time derivatives $\frac{d Y_{i}}{d t}$ and $\frac{d Y_{\text {traced }, i}}{d t}$, this approach requires evaluating the partial derivatives

$$
\begin{gathered}
\frac{\partial}{\partial Y_{j}}\left(\frac{d Y_{i}}{d t}\right), \\
\frac{\partial}{\partial Y_{j}}\left(\frac{d Y_{\text {traced }, i}}{d t}\right), \\
\frac{\partial}{\partial Y_{\text {traced }, j}}\left(\frac{d Y_{i}}{d t}\right), \text { and } \\
\frac{\partial}{\partial Y_{\text {traced }, j}}\left(\frac{d Y_{\text {traced }, i}}{d t}\right) .
\end{gathered}
$$

Insofar as Eqs. 7 and 8 are polynomials of the $Y_{i}$ and $Y_{\text {traced }, i}$, these partial derivatives are straightforward to evaluate, and we do not give their explicit form here.

For the calculations performed in this work, we use PRISM $^{\text {tr }}$ to perform a number of $r$-process nucleosynthesis tracing simulations. In all cases, we implement a combination of experimental data and theory calculations for charged-particle reaction rates; $\beta^{-}$-decay rates; delayed neutron emission probabilities; neutron-capture rates; one-neutron photodissociation rates; neutron-induced, $\beta^{-}$-delayed, and spontaneous fission rates; and fission yields. Charged-particle reaction rate data is taken from the JINA REACLIB database 31. The $\beta^{-}$-decay rates, $\beta^{-}$-delayed fission rates, and delayed neutron emission probabilities are evaluated using the Los Alamos National Laboratory (LANL) QRPA + HF framework of [32, 33] using AME2016 34] and FRDM2012 35] nuclear masses. Neutron-capture rates and neutron-induced fission rates are calculated using the LANL statistical Hauser-Feshbach code $\mathrm{CoH}$ [36], also assuming AME2016 and FRDM2012 nuclear masses. One-neutron photodissociation rates are evaluated by detailed balance, with the requisite one-neutron separation energies taken from the AME2016 and FRDM2012 nuclear masses. Fission yields are taken from the calculations of 37. We implement all decay half-lives and branching ratios of the Nubase 2016 evaluation 38, which are taken to replace the aforementioned theory calculations when possible.

Finally, we note that many of these processes invariably produce one or more free neutrons. We do not, for the present work, intend to trace the nucleosynthesis in which these neutrons participate. In order to prevent such neutrons from populating the traced abundances, we fix $\frac{d Y_{\text {traced,neutron }}}{d t}=0$, instead of evaluating it according to Eq. 8, Future work may investigate, e.g., the relative effect of these neutrons on $r$-process nucleosynthesis, in which case it would be necessary to evaluate $\frac{d Y_{\text {traced,neutron }}}{d t}$ via Eq. 8 


\section{APPLYING THE NUCLEOSYNTHESIS TRACING FRAMEWORK TO THE $r$ PROCESS}

The rapid neutron capture process ( $r$ process) of nucleosynthesis is the astrophysical mechanism by which the heaviest elements observed to exist in the universe are formed. The $r$ process proceeds by an alternating sequence of neutron capture and $\beta^{-}$-decay towards progressively heavier nuclei and is made possible by extremely hot, dense, and neutron-rich environments [1] identifying the astrophysical events that provide such extreme conditions remains one of the greatest open problems in nuclear astrophysics. [39, 40]. Major progress towards this goal occurred with the first gravitational wave observation of a neutron star - neutron star merger, GW170817/GRB170817a/SSS17a [41]. Analysis of the electromagnetic counterpart of this event suggests a significant lanthanide component in the ejecta of this event, pointing to neutron star mergers as one possible site of the $r$ process [42. However, owing to significant challenges in observational astronomy, astrophysics, and nuclear physics, it is not yet possible to clearly identify neutron star mergers as the dominant source of $r$-process nuclei in the universe (see, e.g., [5, 6] and references therein).

The $r$ process poses several barriers to analysis that make it a particularly interesting focus for the first application of our tracing framework. Multiple nuclear processes, including neutron capture, neutron photodissociation, and $\beta^{-}$-decay, are all in competition as thousands of different nuclear species are populated throughout nucleosynthesis; when some of the heaviest and most neutronrich nuclei are formed, fission begins to compete as well, populating lighter nuclei according to complex fission fragment distributions (yields) that potentially span hundreds of different nuclei. Because of the large number of nuclear species involved and the numerous transmutation processes connecting them, it can be especially difficult to quantify how individual transmutation processes interact with the many others to determine the progression of nucleosynthesis.

In this section, we demonstrate several ways that our nucleosynthesis tracing framework may be applied to address the challenges associated with understanding the role of nuclear properties in governing $r$-process nucleosynthesis. Sec. IV A highlights the role of different fission channels - considered as a whole, as well as for individual nuclei - in a variety of neutron star merger $r$ process environments. In Sec. IVB we choose a set of neutron star merger wind conditions where fission plays a minimal role and perform tracing calculations for the $\beta^{-}$-decay of elements $40 \leq Z \leq 80$.

\section{A. Distribution of fission products in final r-process abundances}

In extremely neutron-rich environments, sufficiently heavy nuclei may be formed during the $r$ process such that these nuclei begin to fission, with varying degrees of significance for the nuclear abundances produced from nucleosynthesis. In the most extreme cases, fission recycling may occur, in which the bulk of nuclei undergo fission; these nuclei are returned to lighter nuclei and undergo additional neutron captures and $\beta^{-}$-decays characteristic of the $r$ process. In such conditions, the specific nuclear abundances are expected to depend on the fission properties of many exotic neutron-rich nuclei [16, 17, 4347. However, these fission properties effectively rely entirely on theory-based predictions, and a great deal of progress has been made to evaluate them, including from systematic, macroscopic-microscopic, and purely microscopic theoretical approaches (see, e.g., 48, and references therein for a recent review; also [16, 17, 37, 4952 ). In order to help inform existing and future efforts in the study of nuclear fission, we use our tracing framework to examine the various ways that different fission processes, namely spontaneous fission $(s f), \beta^{-}$-delayed fission $(\beta d f)$, and neutron-induced fission $((n, f))$, can influence $r$-process nucleosynthesis in the ejecta of a neutron star merger.

We begin by considering a parameterized trajectory for the ejecta of a neutron star merger accretion disk wind, with specific entropy $s / k_{B}=40$, timescale $\tau=$ $20 \mathrm{~ms}$, and electron fraction $Y_{e}=0.20$, as used in 30 . Under these conditions, fission plays a subdominant role that is insufficient to be characterized by fission recycling. We perform three separate tracing calculations in which the products of all nuclei which fission via a particular channel, $(n, f), \beta d f$, or $s f$, are followed throughout the remaining nucleosynthesis.

The results of each of these calculations are shown in Fig. 2. The contributions are dominated by the $(n, f)$ and $\beta d f$ channels, with the final distribution of fission products lying in the $80<A<180$ region. Among the fission contributions to the overall pattern, roughly equal contributions arise from the $(n, f)$ and $\beta d f$ channels. In particular, we note that for these conditions, very few nuclei remain to the left of the second $r$-process peak ( $A \sim 130)$ prior to the onset of fission, with contributions to this region being dominated by material that is directly deposited there as fission products. Because this fission occurs relatively late during nucleosynthesis, the material does not significantly move forward into the second or third $(A \sim 195)$ peaks via subsequent neutron capture, as detailed in [16].

We repeat this analysis for dynamical ejecta conditions from the simulations of $[53$. Fission is more significant in this case, with around $60 \%-80 \%$ of heavy nuclei across the entire pattern having participated in fission. Because most (but not all) of the heavy nuclei have been processed through fission, we define this nucleosynthesis to have 


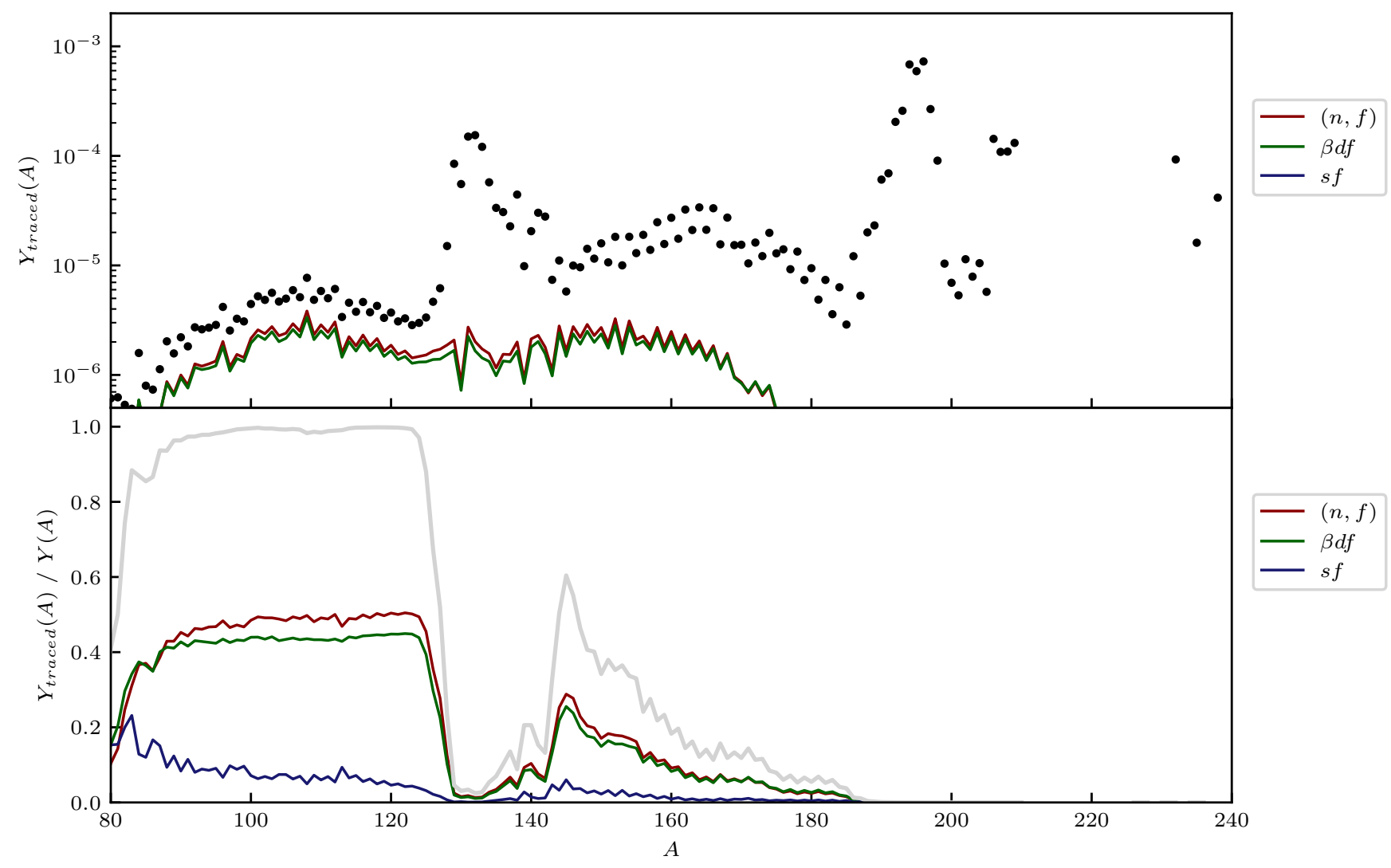

FIG. 2: Relative contributions to final isotopic abundances by terminating fission channel (spontaneous fission, $s f$; neutroninduced fission, $(n, f)$; and $\beta^{-}$-delayed fission, $\left.\beta d f\right)$ for the neutron star merger wind conditions described in the text. The top panel compares the traced abundances (solid lines) to the total abundances (dots). The bottom panel shows the ratio of each traced abundance to the total abundance; the gray line indicates their sum. For $A<125$, all abundances are populated almost exclusively by fission processes, while the relative contributions to $A>125$ are comparatively weaker, peaking at $\sim 50 \%$.

proceeded via incomplete fission recycling.

Significant amounts of $(n, f)$ products undergo followup neutron-capture nucleosynthesis, forming up to $60 \%$ of abundances well beyond the extent of the fission yields, including the third $r$-process peak and long-lived actinide isotopes. These contributions arise from earlier stages of nucleosynthesis, when free neutrons are still relatively abundant and extremely neutron-rich nuclei along the $r$ process path undergo $(n, f)$.

Nuclei to the left of the second peak are populated via a mechanism similar to that of the wind conditions from Fig. 2. Towards the end of nucleosynthesis, the free neutron abundance is sufficiently low such that further neutron capture does not significantly occur following fission. Because of this, significant contributions in the $80<A<125$ region arise from the late-time $(n, f)$ and $\beta d f$ of less neutron-rich nuclei.

Finally, we consider cold tidal-tail conditions from 53 . Here, all heavy nuclei have been processed one or more times through fission, possibly via multiple fission channels. For our analysis, we consider only contributions that arise from the terminating, or last, fission event. In order to achieve this, we trace-in all fission events for the particular channel under consideration and trace-out all other fission events. If a particular abundance in the final pattern has a history involving two different fission channels, only the contribution from the last fission event is considered. As can be seen in Fig. 4, the effect of this is that the sum of fission traces across the $(n, f), \beta d f$, and $s f$ channels add neatly to unity, even though the average nucleus has more than one fission event in its history. Insofar as all heavy nuclei have been processed at least once through fission, we define this nucleosynthesis to have proceeded via complete fission recycling.

In contrast with the calculations shown in Fig. 3. $\beta d f$ can become active earlier in nucleosynthesis, in competition with $(n, f)$ for nuclei along the $r$-process path. As evidence of this, note that the $\beta d f$ products are able to undergo further neutron capture reactions, eventually populating $\sim 10 \%$ of the third peak and long-lived actinide abundances, in addition to some movement of the products from the $A<125$ region into the second peak. As with the previous conditions, nuclei to the left of the second peak are dominated by late-time fission products, 


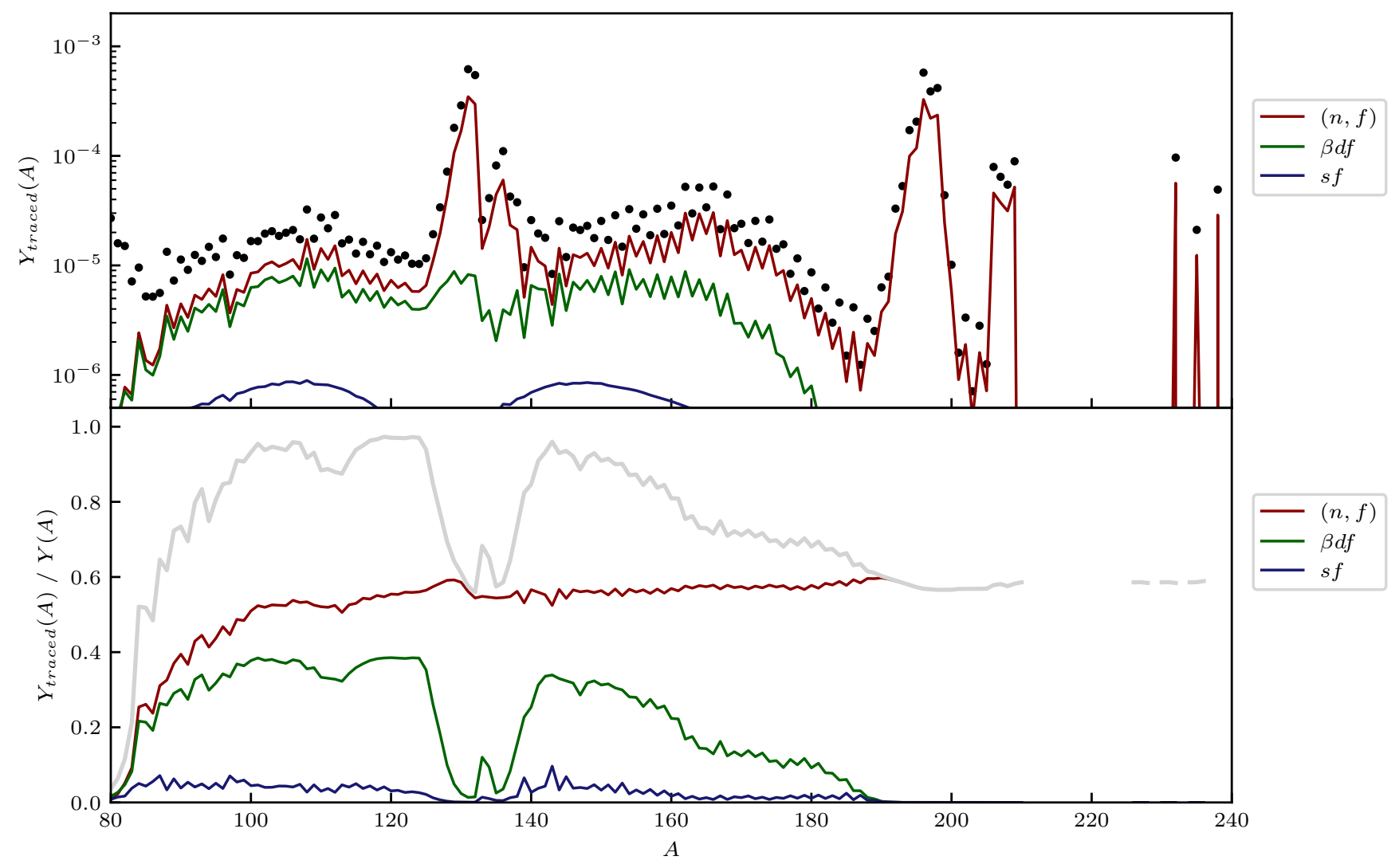

FIG. 3: Relative contributions to final isotopic abundances by terminating fission channel (spontaneous fission, $s f$; neutroninduced fission, $(n, f)$; and $\beta^{-}$-delayed fission, $\left.\beta d f\right)$ for dynamical ejecta conditions from a neutron star merger simulation [53], as in Fig. 2 For these conditions, nucleosynthesis proceeds via incomplete fission recycling, with neutron-induced fission accounting for $\sim 60 \%$ of abundances across the entire range of the pattern, and $\beta^{-}$-delayed fission accounting for an additional $10 \%$ to $30 \%$ for $A<\sim 180$. The remaining abundances, about $10 \%$ to $40 \%$ depending on $A$, have no history of fission.

with roughly equal contributions from the $(n, f)$ and $\beta d f$ channels.

While valuable insight can be derived from tracing entire fission channels across all nuclei, it is also possible to apply our tracing technique with much finer resolution by tracing the fission of individual nuclei. While integrated fission flows have helped inform which nuclei most actively undergo fission during the $r$ process (see e.g. [16, 17]), such approaches provide limited information relating to the distribution of the fission products throughout the abundance pattern at the conclusion of nucleosynthesis. These effects become particularly important in conditions for which nucleosynthesis proceeds via complete or incomplete fission recycling, where there is a combination of early-time fission (whose products are significantly reprocessed via neutron capture), latetime fission (whose products are mostly restricted to $\beta^{-}$decays toward stable nuclei), and intermediate cases.

We perform tracing calculations for the $(n, f)$ and $\beta d f$ of each nuclide found to fission in the cold tidal-tail ejecta considered in Fig. 4 and appurtenant discussion. While $\sim 300$ nuclides fission via either channel during nucle- osynthesis, we find that the overall contribution of their products to the final calculated abundances is quite small for the majority of these, on the order of $1 \%$ or less. By restricting to fission processes with traced abundances constituting a minimum of $10 \%$ of the final pattern for at least one value of $A$, we find 9 fission processes to be significant in these conditions, distributed across very neutron-rich neptunium $(\mathrm{Z}=93)$ and plutonium $(\mathrm{Z}=94)$ isotopes. Collectively, these drive the effects of early-time fission events presented in the discussion of Fig. 4.

In Fig. 5. we plot the traced abundances for each of these 9 fission processes. In each case, the traced abundances follow the same shape as the total pattern, suggesting that the fission products from these nuclei do not imprint on the final abundance pattern, instead quickly reequilibrating along the $r$-process path - an interpretation that is consistent with that of Fig. 4. To reinforce this point, we compare the actual fission yield with the traced abundances for the most-significantly fissioning $(n, f)$ nuclide, neptunium-290, in Fig. 6. While the fission yield is smoothly distributed along $90<A<190$, the products are eventually redistributed throughout the 


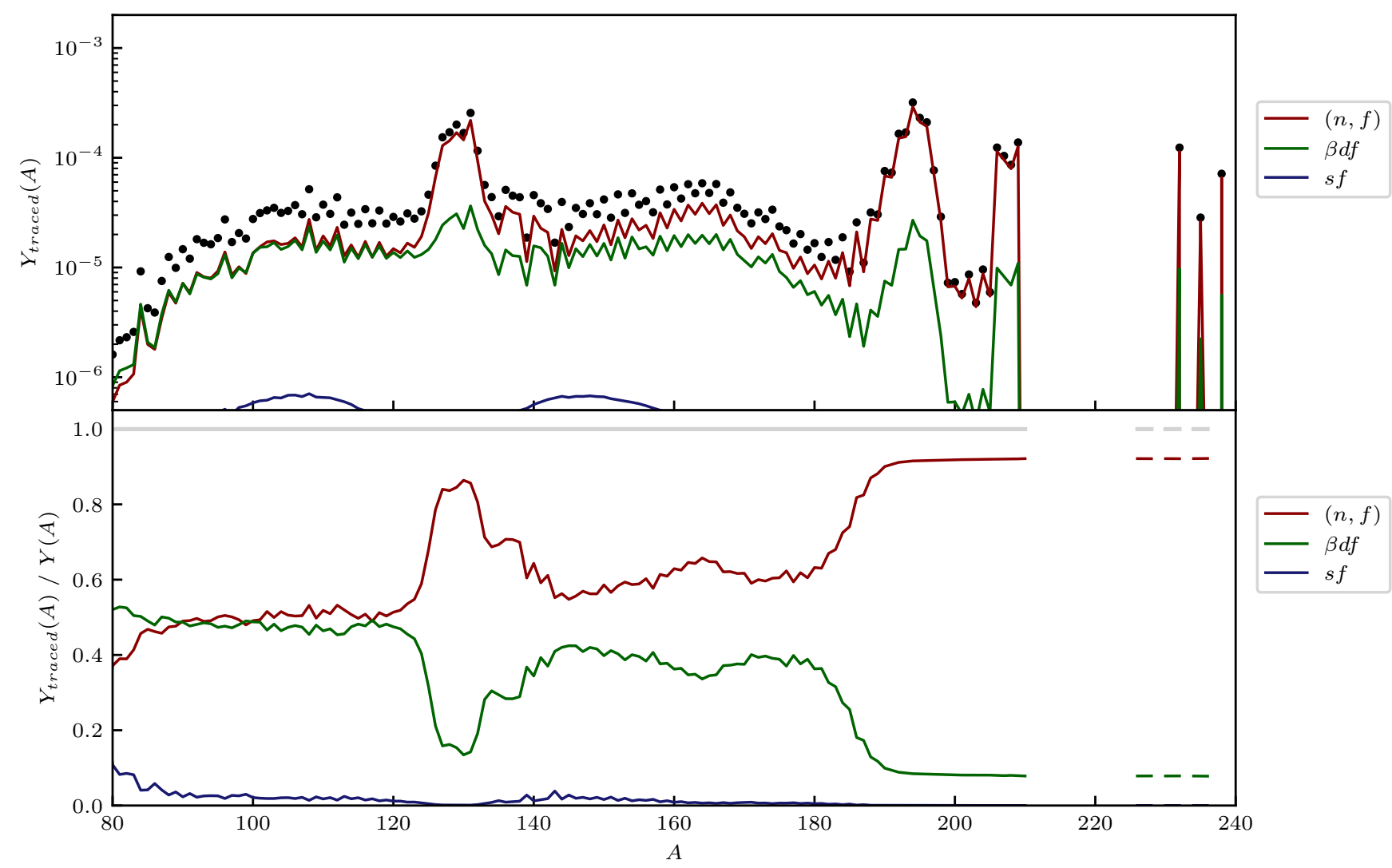

FIG. 4: Relative contributions to final isotopic abundances by terminating fission channel (spontaneous fission, $s f$; neutroninduced fission, $(n, f)$; and $\beta^{-}$-delayed fission, $\left.\beta d f\right)$ for the cold dynamical ejecta conditions from a neutron star merger simulation [53, as in Fig. 2 and Fig. 3 Under these conditions, nucleosynthesis proceeds via complete fission recycling, with $100 \%$ of the pattern having a traced history involving $(n, f), \beta d f$, or $s f$.

second and third $r$-process peaks and long-lived actinide isotopes, in proportion to the total abundance pattern.

Some fraction of material processed through these $\sim 9$ early-time fission processes will eventually undergo a final late-time fission event sometime after the free neutron abundance begins to subside. Consequently, nuclei will be distributed according to the yields of these final fission events without significant reprocessing by neutron capture. Because these calculations continue to trace fission products through all subsequent fission events, we see the formation of the $80<A<125$ abundances in Fig. 5. Our tracing calculations suggest that, for an $r$ process proceeding via complete fission recycling, abundance features which eventually form via late-time fission were first processed through the fission of just a handful of nuclear species, in this particular case the $\sim 9$ fission processes we have identified here.

If we consider nuclei that fission below the $10 \%$ threshold used in the preceding discussion, we find a large number of late-time fission processes whose yields leave a static imprint on the total abundance pattern. In Fig. 7 . we compare the fission yield to the traced abundances for one such example, the late-time $\beta d f$ of berkelium-270 $(\mathrm{Z}=97)$. The traced abundances, in this case, clearly follow the fission yield, with any discrepancies arising from $\beta^{-}$-delayed neutron emission that happens as the products decay towards stable nuclei, effectively shifting some of the products toward lower values of $A$.

Figure 8 places the $(n, f)$ and $\beta d f$ of each nuclide into the dichotomy of pattern-like (as in Fig. 6) and yield-like (as in Fig. 7) traced abundances. We begin by calculating integrated fission flows, defined for each nuclide $i$ as $\int \Lambda_{(n, f), i} Y_{n} Y_{i} d t$ and $\int \Lambda_{\beta d f, i} Y_{i} d t$ for $(n, f)$ and $\beta d f$, respectively; here, $\Lambda$ is as defined in Sec. II. For every fission process with an integrated fission flow in excess of $10^{-7}$, we evaluate the functions

$$
\begin{gathered}
L_{\text {pattern }}=\frac{1}{2} \sum_{A>80}\left|Y(A)-Y_{\text {traced }}(A)\right| \\
L_{\text {yield }}=\frac{1}{2} \sum_{A>80}\left|\operatorname{Yield}(A)-Y_{\text {traced }}(A)\right|
\end{gathered}
$$

where $Y(A), Y_{\text {traced }}(A)$, and $\operatorname{Yield}(A)$ are the total abundances, traced abundances, and fission yields, respec- 


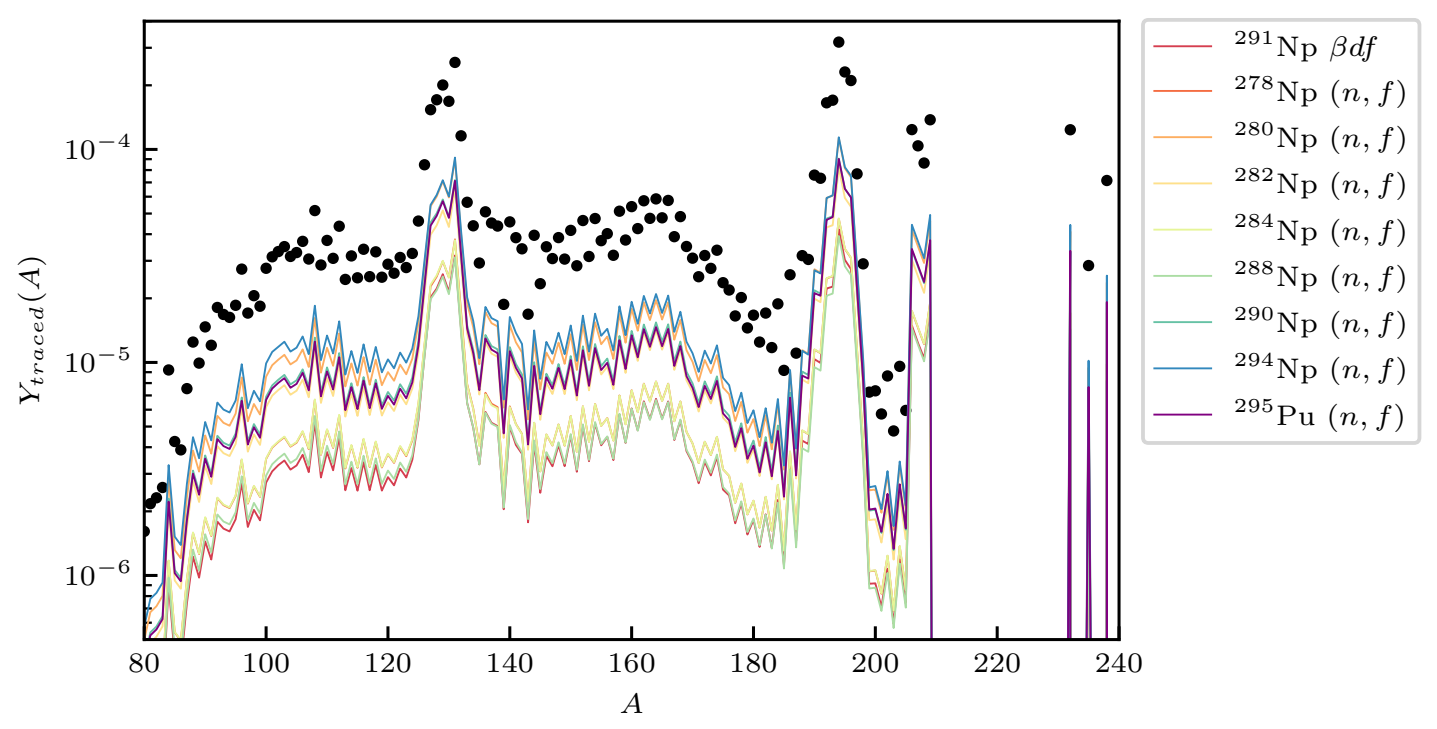

FIG. 5: Relative contributions to final isotopic abundances, $\left(Y(A)\right.$, solid dots) for the $\beta^{-}$-delayed $(\beta d f)$ and neutron-induced $((n, f))$ fission products of individual neptunium and plutonium isotopes (solid lines) in the cold dynamical ejecta conditions of [53], as in Fig. 4. The plotted isotopes are those whose fission yields contribute at least $10 \%$ to the total abundance pattern for at least one mass number $A$.

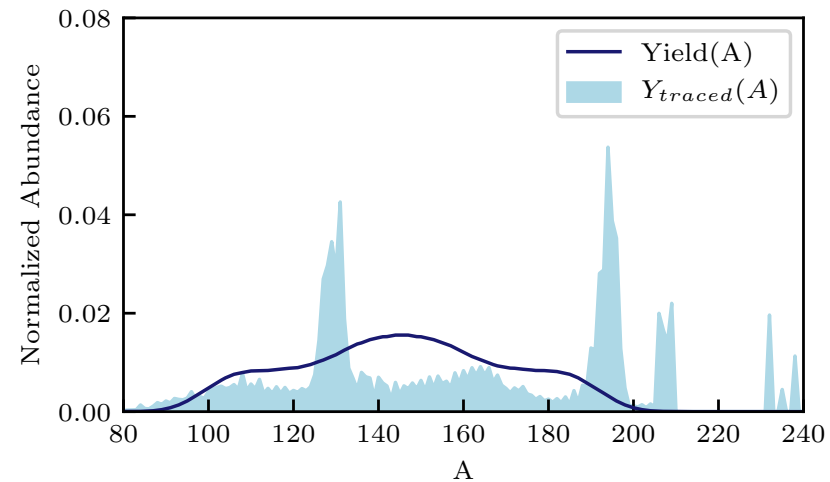

FIG. 6: Comparison of fission yield (solid line) to final traced abundances (shaded region) for the neutron-induced fission of neptunium-290 in the cold dynamical ejecta conditions of [53], as in Fig. 4 Both are normalized according to Eq. 11. Nuclei produced by this fission process participate in significant further neutron-capture nucleosynthesis, with the actual fission yield leaving a minimal imprint on the final abundance pattern.

tively, and each is normalized such that

$$
\begin{aligned}
& \sum_{A>80} Y(A)=1, \\
& \sum_{A>80} \operatorname{Yield}(A)=1, \text { and } \\
& \sum_{A>80} Y_{\text {traced }}(A)=1 .
\end{aligned}
$$

In this way, $L_{\text {yield }}$ is nearly zero if the traced abundances follow the same distribution as the corresponding fission yield. Likewise, $L_{\text {pattern }}$ is nearly zero if the distribution of the traced abundances follows that of the total abundances. By comparing $L_{\text {yield }}$ to $L_{\text {pattern }}$, we may systematically identify whether the $(n, f)$ and $\beta d f$ of each nuclide is pattern-like or yield-like. In Fig. 8 , the $(n, f)$ and $\beta d f$ traced abundances for each nuclide are colored red if they are yield-like and blue if pattern-like, and the shading of each indicates integrated fission flow.

Along the $r$-process path ( $Z \sim 95$ and $N \geq 185$ ), all of the traced abundances are pattern-like, suggesting that these fission products quickly reequilibrate along the existing $r$-process path. For the remaining less neutron-rich nuclides, the traced abundances are consistently yieldlike, and their contribution to the overall isotopic abundances are mostly in proportion to their respective fission yields.

The yield-like nuclides for $\beta d f$ and $(n, f)$ are distributed over a relatively large number of nuclides. Collectively, their fission products play a significant role in shaping certain features of the final abundance pattern, consistent with the interpretation of results presented in Fig. 2, Fig. 3, and Fig. 4. On the other hand, the effects of individual fission yields are averaged out across these many different nuclides. Indeed, in no case do any of the yield-like traced abundances constitute more than $\sim 7 \%$ of the total abundances for any value of $A$. While the early-time fission of the most neutron-rich nuclei tends to be more significantly focused on only a few nuclei, these contributions tend to be pattern-like and, therefore, similarly insensitive to fine details the associated yields. 
Instead, the final nucleosynthetic outcome is shaped by average trends in fission yields for the many nuclei populated during the decay to stability at late stages of the $r$ process, consistent with the results of, e.g., [45. While these conclusions are drawn from a specific set of nuclear data, and as shown e.g. in [17] different sets of data will lead to distinct predictions for impactful fissioning nuclei, we expect these general trends to hold.

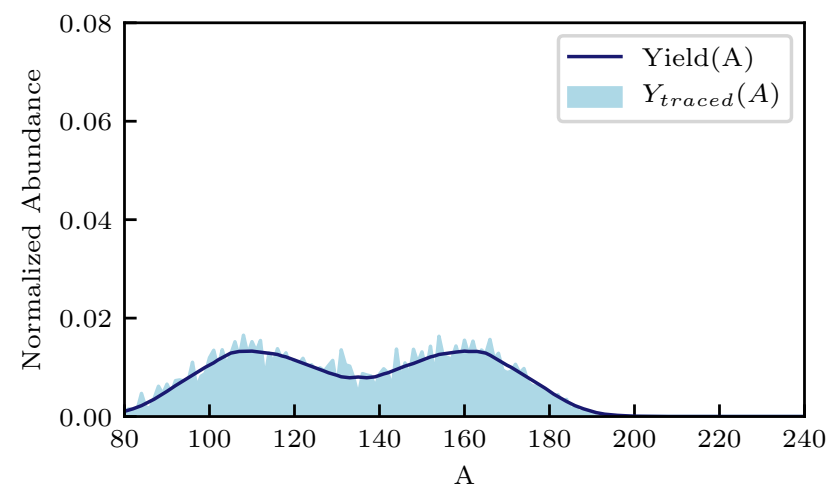

FIG. 7: Comparison of fission yield (solid line) to final traced abundances (shaded region) for the $\beta^{-}$-delayed fission of berkelium-270, as in Fig. 6. Nuclei produced by this fission process primarily undergo a series of $\beta^{-}$-decays, with minimal effect on the distribution in mass number, $A$, compared to that of the original yield.

\section{B. Tracing $\beta^{-}$-decays in an $r$ process}

Because the $r$ process involves the most neutron-rich nuclei, many of these nuclei are difficult to study experimentally, and nucleosynthesis simulations rely heavily on predictions from theoretical nuclear models. Beyond the limits of experimental data, theoretical predictions for these nuclei diverge [54 56], introducing a significant source of uncertainty in $r$ process nucleosynthesis simulations [9 11, 14. Experimental campaigns at current and upcoming facilities such as CARIBU [57 62] and the $N=126$ Factory [63] at ATLAS, IGISOL at Jyväskylä 64, 65, ISOLDE at CERN 66, TITAN at TRIUMF [67], RIKEN 68 70, GSI/FAIR 7173], and FRIB [4] are approaching nuclei of interest to the $r$ process.

In this context, it will be especially important to identify which nuclei are of critical importance to understanding and constraining $r$-process nucleosynthesis simulations. Here, we demonstrate how our nucleosynthesis tracing technique can help to accomplish this task. We focus specifically on only one category of nuclear data on which $r$ process nucleosynthesis simulations critically depend, namely $\beta^{-}$-decay properties for neutron-rich nuclei. Nuclear $\beta^{-}$-decay is the transmutation process responsible for moving neutron rich nuclei towards heavier elements during the $r$ process. In addition to controlling the number retained at 'waiting points' associated with closed neutron shells, $\beta^{-}$-decay can also compete with $(n, \gamma)$ and $(\gamma, n)$ reactions to adjust the nuclear abundances produced during the $r$ process for as long as these reaction channels remain active (see, e.g., [15, 75, 79]). We focus on identifying which of these $\beta^{-}$-decays an $r$ process most strongly depends.

To simplify the interpretation of our results, we select a parameterized neutron star merger wind in which fission does not participate as an active process during nucleosynthesis, with parameters $s / k_{B}=50, \tau=50 \mathrm{~ms}$, and $Y_{e}=0.25$. For each nuclide with $40 \leq Z \leq 80$ populated at any point during nucleosynthesis, we perform a tracing calculation for its $\beta^{-}$-decay. The resulting calculation indicates the relative fraction of each abundance with a history involving the $\beta^{-}$-decay under consideration.

The traced $\beta^{-}$-decays can be roughly sorted into three distinct, yet physically intuitive, categories. For nuclides nearest stability, their $\beta^{-}$-decays occur well after the free neutron abundance has been exhausted, and so these preserve the mass number $A$ with respect to the final pattern. Along the $r$-process path, the most neutron-rich isotopes populated during an $r$ process, the bulk of of all heavier nuclei will proceed through these nuclei via $\beta^{-}$decay. As a result, the traced abundances will reproduce nearly the entire pattern for all larger values of $A$. Finally, one can imagine an intermediate case, where nuclei begin to fall back towards stability as a result of decreasing free neutron abundances but may still participate in some degree of neutron capture. In Fig. 9, we show an example of each regime for a selection of neodymium $(Z=60)$ isotopes. In the case of neodymium-152, all of the abundances in the final overall pattern have participated in this $\beta^{-}$-decay while decaying back to stability after the completion of the $r$ process. For neodymium-186, which lies along the $r$ process path, the majority of populated nuclear species with $A \geq 186$ have participated in this particular $\beta^{-}$-decay. Finally, we highlight the intermediate case with neodymium-176, lying between stability and the $r$-process path, where some fraction of abundances for multiple nearby $A$ have a history involving this $\beta^{-}$-decay.

In Fig. 10, we quantify average trends that arise in these tracing network calculations. For each traced pattern, we define the set

$$
W=\left\{A^{\prime}>A \mid Y_{\text {traced }}\left(A^{\prime}\right) / Y\left(A^{\prime}\right)>1 \%\right\}
$$

where $A$ is the mass number of the traced $\beta^{-}$-decay parent nucleus. The set $W$ represents the values of $A$ for which the traced abundance represent at least $1 \%$ of the final abundance. We refer to the cardinality of the set $W$ as the width of the traced pattern. By only considering $A^{\prime}>A$, we omit any contributions to the final pattern that occur during decay back to stability following the $r$ process. As a result, the width is restricted to contributions that are dynamically involved in the $r$ process.

In the top panel of Fig. 10, we report the width of 

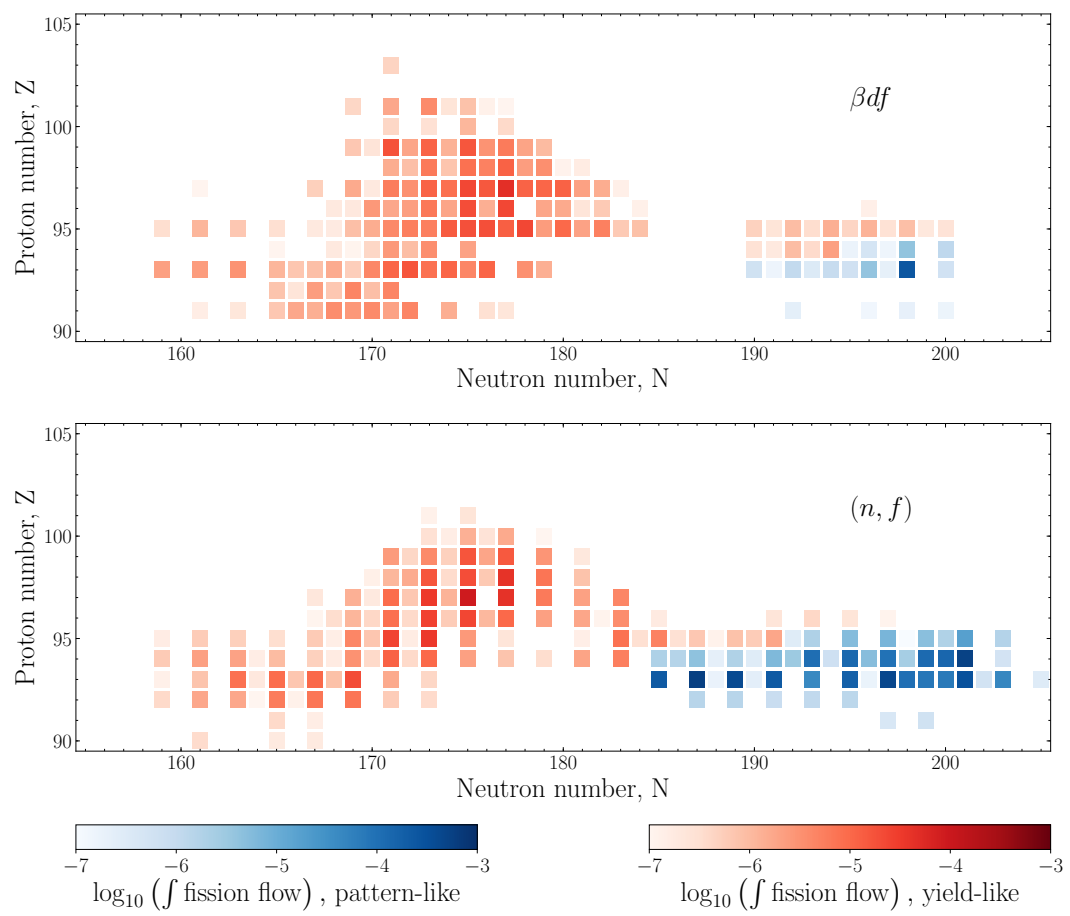

FIG. 8: Integrated $\beta$-delayed ( $\beta d f$, top panel) and neutron-induced $((n, f)$, bottom panel) fission flows for individual nuclides during the cold dynamical ejecta conditions of [53, as in Fig. 4 Red color indicates that the traced isotopic abundances are mostly similar to the fission yield $\left(Y_{\text {traced }}(A) \sim \operatorname{Yield}(A)\right.$, as in Fig. 7), and blue color indicates that the traced isotopic abundances are similar to the overall pattern $\left(Y_{\text {traced }}(A) \sim Y(A)\right.$, as in Fig. 6.

each of our tracing calculations. For each element, the width is greatest along the $r$-process path, since the vast majority of abundances of heavier nuclides are produced along this path. Near stability, the width collapses to 0 because all subsequent nucleosynthesis strictly follows a series of $\beta^{-}$-decays that preserve mass number $A$, which is omitted from the set $W$ as we have constructed it. In the intermediate region, there is a smooth transition from larger to smaller widths, with values ranging from 2-10 for a relatively large number of nuclides lying away from the $r$ process path.

It is also instructive to consider the average contribution of a particular $\beta^{-}$-decay to the total abundance pattern. We define an additional metric that attempts to provide this insight, defined as

$$
H_{\text {avg. }}=\frac{1}{|W|} \cdot \sum_{A^{\prime} \in W} Y_{\text {traced }}\left(A^{\prime}\right) / Y\left(A^{\prime}\right)
$$

where $W$ is the same as in Eq. 12 and $|W|$ is the width. The value of $H_{\text {avg. }}$ can be understood as the relative contribution of a particular $\beta^{-}$-decay, on average, to nuclides contained within the width of the contribution. A large value represents significant contributions to the entirety of the width of the traced pattern, while smaller values correspond to less significant contributions.
Analogous to this metric is the summed relative contributions, given by

$$
H_{\text {sum }}=\sum_{A^{\prime} \in W} Y_{\text {traced }}\left(A^{\prime}\right) / Y\left(A^{\prime}\right)
$$

Large values in this metric indicate a relatively large width together with significant contributions to overall abundances to the same width.

The values of $H_{\text {avg. }}$ and $H_{\text {sum }}$ are shown in the middle and bottom panels of Fig. 10, respectively. As with the widths shown in the top panel, the largest values in each metric lie along the path for the same reasons previously discussed. However, in the intermediate region lying between the $r$-process path and decay-to-stability nuclides, we can further constrain the list of 'impactful' $\beta^{-}$-decays for these conditions. Many of the $\beta^{-}$-decays with relatively wide contributions to the abundance pattern have comparatively weak contributions, less than $10 \%$, and may reasonably be considered less important in determining the final abundances overall.

As can be observed in the bottom panel, $\beta^{-}$-decay for nuclides nearer the $r$-process path have sufficiently wide contributions affecting a larger region of the abundance pattern. For nuclides in the intermediate region but nearer stability, there can be still-significant contributions, but these contributions are focused on more con- 


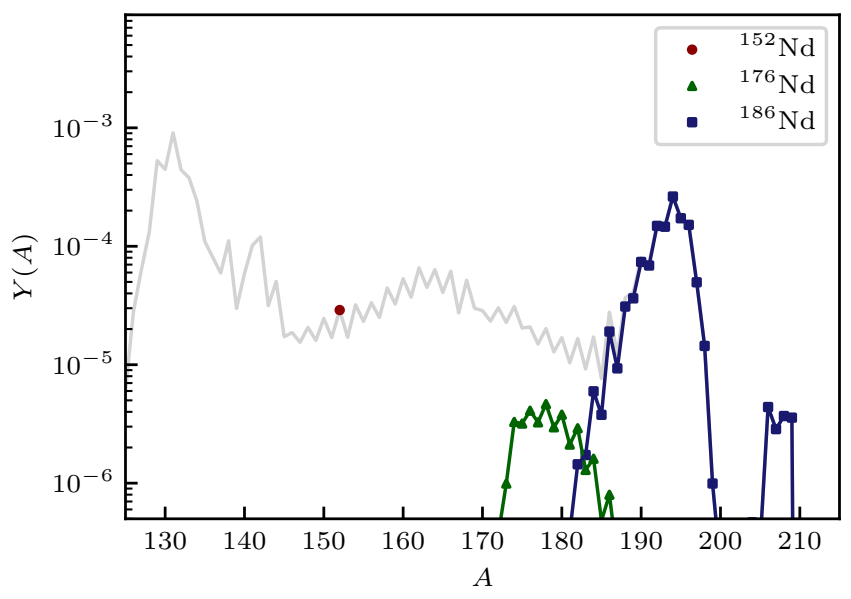

FIG. 9: Traced isotopic abundances for the $\beta^{-}$-decay of a selection of neodymium isotopes (neodymium-152, red circle; neodymium-176, green triangles; and neodymium-186, blue squares). The total abundances are given by the gray line for comparison. Neodymium-152 is populated as nuclei are decaying to stable nuclei, and the entirety of abundances for $A=152$ have undergone this $\beta^{-}$-decay. Neodymium-186 lies on the $r$-process path, and all nuclei with $A \geq 186$ have undergone this $\beta^{-}$-decay. Neodymium-176 represents an intermediate case, where some fraction, $\sim 10 \%$, of nuclei with $A \sim 176$ having been produced by this $\beta^{-}$-decay.

strained regions of the abundance pattern, as they have a significant value for $H_{\text {avg. }}$ but smaller values for the width and, consequently, $H_{\text {sum }}$.

Clearly, $\beta^{-}$-decay rates along the $r$-process path dominate all three of the metrics considered in this section. However, by applying the nucleosynthesis tracing framework, it becomes clear that a large number of $\beta^{-}$-decay rates for less neutron-rich nuclides can also significantly influence nucleosynthesis in $r$-process environments.

As a final caution, we point out that these results strongly depend on the astrophysical conditions and the theoretical nuclear models used to supplement available experimental data. Changes to either are liable to affect the $r$-process path and the onset of fission recycling, in addition to other possible complications. We propose here only the method by which more robust analyses may proceed in future works. However, we do anticipate the general result to hold; namely, $\beta^{-}$-decay rates of many nuclides less neutron-rich than the $r$-process path are still important in determining nucleosynthesis. We emphasize the importance of future experiments that measure the $\beta^{-}$-decay rates (or other properties, such as nuclear masses) for these nuclides, even if the most neutron-rich nuclei remain out of reach for the foreseeable future.

\section{CONCLUSION}

The most complex examples of nucleosynthesis involve thousands of nuclear species connected by many tens of thousands of nuclear transmutation processes. Owing partly to this complexity, as well as to the generally dynamic and nonlinear nature of nucleosynthesis, it is often difficult to study subsets of nuclear properties in isolation from a nucleosynthetic system as a whole. In this work, we develop our nucleosynthesis tracing framework, which may be applied to partly address this problem.

Beginning with the system of coupled differential equations constituting a standard nuclear reaction network, we frame our tracing framework as the separation of nuclear abundances into two populations, those of traced and untraced abundances. Furthermore, we allow each transmutation process in a network calculation to be categorized by the way it maps reactants and products between the traced and untraced populations. Within this schema, we derive an additional set of differential equations that model the evolution of the traced abundances. These additional equations are coupled to those of the standard network; when solved together, one obtains a quantitative description of how products from specific nuclear transmutation processes participate in all subsequent nucleosynthesis.

We implement our tracing framework into a new version of our PRISM reaction network, PRISM ${ }^{\text {tr }}$, and comment on several details regarding this implementation. Notably, the tracing network equations are structurally similar to those of a standard reaction network; therefore, numerical techniques commonly used to solve the standard set of network equations are expected to be equally well-suited for solving the tracing network equations.

In order to demonstrate some of the nucleosynthesis analyses enabled by our tracing framework, we perform tracing network calculations using PRISM ${ }^{\text {tr }}$ to study fission and $\beta^{-}$-decay as they occur in the $r$ process of nucleosynthesis.

Our application of tracing to distinct fission channels can quantify the influence of each channel on forming the final abundance pattern, with qualitative results consistent with investigations of fission in the $r$ process found in the literature. These same calculations offer insight into the extent of fission recycling in an $r$-process simulation -in particular, they allow for the quantitative distinction between complete and incomplete fission recycling in the $r$ process.

When the tracing framework is applied to individual fission reactions/decays, we find the fission of a relatively small number of nuclear species along the $r$-process path drives fission recycling. The fission yields of these nuclei have limited impact on the final abundance pattern since the fission products undergo subsequent neutron captures and are redistributed throughout the network. The shape of the final abundance pattern is instead determined by the product yields of the many nuclear species that fission as the $r$-process path moves back to stabil- 

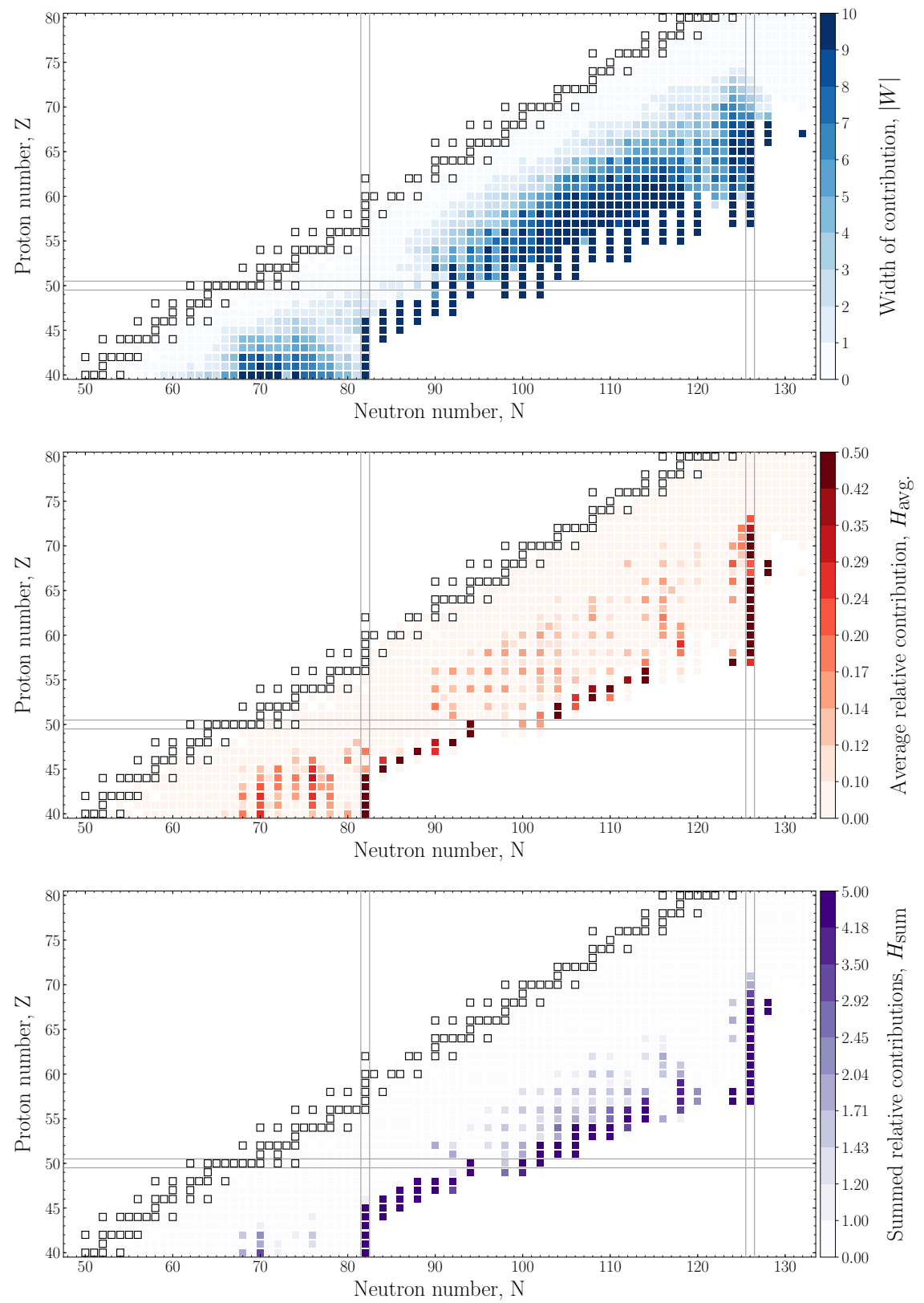

FIG. 10: Average trends in the traced abundances of individual $\beta^{-}$-decays for elements $40<Z<80$. The top panel shows the 'width' $(|W|)$ of the traced abundances, as defined in the text. The middle panel indicates the average relative contribution to total abundances contained within the width $\left(H_{\text {avg. }}\right)$. The bottom panel gives the the sum of relative contributions to the width $\left(H_{\text {sum }}\right)$, which may be interpreted as the product of the top and middle panels.

ity upon neutron exhaustion. Thus, average trends in fission yields for a large number of nuclei are needed to characterize $r$-process nucleosynthesis.

Additionally, we apply nucleosynthesis tracing to perform a comprehensive examination of the $\beta^{-}$-decay of nuclei with atomic number $40 \leq Z \leq 80$. We quantify the relative contribution of each $\beta^{-}$-decay to $r$-process nucleosynthesis in neutron star merger wind-like conditions, and we define several metrics that may be useful for characterizing the nature of these contributions.
Finally, we strongly emphasize that our fission and $\beta^{-}$decay results depend on the astrophysical conditions and underlying nuclear models used for this study; a thorough investigation of these dependencies, together with a more comprehensive examination of the numerous and varied nuclear properties entering into $r$-process nucleosynthesis calculations, is intended for future work.

While we limit the present work to $r$-process applications, we note that our tracing framework - as we have presented it -is in no way limited to $r$-process nucle- 
osynthesis, and it may be readily applied to any process for which nuclear reaction networks are appropriate. Indeed, the defining principles of our tracing framework can be naturally adapted to applications outside of nucleosynthesis entirely, e.g., chemical reaction networks.

\section{ACKNOWLEDGEMENTS}

This work was supported in part by the US Department of Energy under Contract No. DE-FG02-95ER40934, the topical collaboration Fission In R-process Elements (FIRE) Contract No. DE-AC52-07NA27344, and SciDAC Contract No. DE-SC0018232. MM was supported by the US Department of Energy through Los Alamos National Laboratory and by the Laboratory Directed Research and Development program of Los Alamos National Laboratory under project number 20190021DR. Los Alamos National Laboratory is operated by Triad National Security, LLC, for the National Nuclear Security Administration of U.S. Department of Energy (Contract No. 89233218CNA000001). T.S. was supported in part by the Los Alamos National Laboratory Center for Space and Earth Science, which is funded by its Laboratory Directed Research and Development program under project number 20180475DR.

\section{Appendix: Some comments on our reaction network formalism}

The notation we adopt in our construction of the nuclear reaction network equations was chosen to simplify the expressions used in the derivation and statement of the tracing network equations. However, this notation differs from more commonly adopted forms, such as that used in [18]. Here, we relate our notation to this more common version.

Beginning with Eq. 12 from [18, the time derivative $\dot{Y}_{i}$ of each nuclear abundance $Y_{i}$ is given by

$$
\begin{aligned}
\dot{Y}_{i} & =\sum_{j} \mathcal{N}_{j}^{i} \lambda_{j} Y_{j}+\sum_{j, k} \mathcal{N}_{j, k}^{i} \rho N_{A}\langle j, k\rangle Y_{j} Y_{k} \\
& +\sum_{j, k, l} \mathcal{N}_{j, k, l}^{i} \rho^{2} N_{A}{ }^{2}\langle j, k, l\rangle Y_{j} Y_{k} Y_{l},
\end{aligned}
$$

where each sum is taken over the one-, two-, and threebody reactions in which species $i$ is either created or destroyed. Here, $\lambda_{j}$ is a decay rate for species $j, \rho$ is the density, $N_{A}$ is Avogadro's number, $\langle j, k\rangle$ is the thermal reaction cross section for a reaction between species $j$ and $k$, and $\langle j, k, l\rangle$ is the thermal cross section for a reaction between species $j, k$, and $l$. The $\mathcal{N}_{j}^{i}, \mathcal{N}_{j, k}^{i}$, and $\mathcal{N}_{j, k, l}^{i}$ are numerical factors that correctly count the number of species consumed or produced in each reaction, defined as

$$
\begin{aligned}
\mathcal{N}_{j}^{i} & =N_{i} \\
\mathcal{N}_{j, k}^{i} & =N_{i} / \prod_{m=1}^{n_{m}}\left|N_{j_{m}}\right| !, \text { and } \\
\mathcal{N}_{j, k, l}^{i} & =N_{i} / \prod_{m=1}^{n_{m}}\left|N_{j_{m}}\right| !,
\end{aligned}
$$

with $N_{i}$ giving the number of species $i$ produced (positive) or consumed (negative) by a reaction or decay, and the product in the denominator run over all species consumed by a reaction and corrects for overcounting a reaction involving identical reactants.

In relation to the terminology and notation we use in Sec. II, each term in Eq. A.1 corresponds to a unique process, $p$, in a network. The processes are grouped into one-, two-, and three-body processes in the first, second, and third sums, respectively. In the case of one-body process, then, we have the associations

- $\Lambda_{p}=\lambda_{j}$

- $\alpha_{p}(i)=1$ if $i$ is a reactant of $p$ and 0 otherwise, and

- $\beta_{p}(i)=\left|N_{i}\right|$ if $i$ is a product of $p$ and 0 otherwise.

For two-body processes, the associations are given by

- $\Lambda_{p}=\rho N_{A}\langle j, k\rangle \cdot \frac{\mathcal{N}_{j, k}^{i}}{N_{i}}$,

- $\alpha_{p}(i)=\left|N_{i}\right|$ if $i$ is a reactant of $p$ and 0 otherwise, and

- $\beta_{p}(i)=\left|N_{i}\right|$ if $i$ is a product of $p$ and 0 otherwise.

Finally, for three-body processes, we have

- $\Lambda_{p}=\rho^{2} N_{A}^{2}\langle j, k, l\rangle \cdot \frac{\mathcal{N}_{j, k, l}^{i}}{N_{i}}$,

- $\alpha_{p}(i)=\left|N_{i}\right|$ if $i$ is a reactant of $p$ and 0 otherwise, and

- $\beta_{p}(i)=\left|N_{i}\right|$ if $i$ is a product of $p$ and 0 otherwise.

In all cases, the sets $\mathcal{R}_{p}$ and $\mathcal{P}_{p}$ simply collect the reactants and products of the process $p$, which we use to make explicit the limits of the summations and products used in Eq. 1

By writing the one-, two-, and three-body terms separately, collecting the positive and negative terms of each into a common summation, and performing the substitutions defined above, Eq. 1 can be rewritten as Eq. A.1. 
[1] E. Margaret Burbidge, G. R. Burbidge, William A. Fowler, and F. Hoyle. Synthesis of the Elements in Stars. Reviews of Modern Physics, 29(4):547-650, January 1957.

[2] George Wallerstein, Jr. Iben, Icko, Peter Parker, Ann Merchant Boesgaard, Gerald M. Hale, Arthur E. Champagne, Charles A. Barnes, Franz Käppeler, Verne V. Smith, Robert D. Hoffman, Frank X. Timmes, Chris Sneden, Richard N. Boyd, Bradley S. Meyer, and David L. Lambert. Synthesis of the elements in stars: forty years of progress. Reviews of Modern Physics, 69(4):995-1084, October 1997.

[3] C. A. Bertulani and A. Gade. Nuclear astrophysics with radioactive beams. Physics Reports, 485(6):195-259, January 2010.

[4] Thomas Rauscher. The Path to Improved Reaction Rates for Astrophysics. International Journal of Modern Physics E, 20(5):1071-1169, January 2011.

[5] C. J. Horowitz, A. Arcones, B. Côté, I. Dillmann, W. Nazarewicz, I. U. Roederer, H. Schatz, A. Aprahamian, D. Atanasov, A. Bauswein, T. C. Beers, J. Bliss, M. Brodeur, J. A. Clark, A. Frebel, F. Foucart, C. J. Hansen, O. Just, A. Kankainen, G. C. McLaughlin, J. M. Kelly, S. N. Liddick, D. M. Lee, J. Lippuner, D. Martin, J. Mendoza-Temis, B. D. Metzger, M. R. Mumpower, G. Perdikakis, J. Pereira, B. W. O'Shea, R. Reifarth, A. M. Rogers, D. M. Siegel, A. Spyrou, R. Surman, X. Tang, T. Uesaka, and M. Wang. r-process nucleosynthesis: connecting rare-isotope beam facilities with the cosmos. Journal of Physics G Nuclear Physics, 46(8):083001, Aug 2019.

[6] T. Kajino, W. Aoki, A. B. Balantekin, R. Diehl, M. A. Famiano, and G. J. Mathews. Current status of rprocess nucleosynthesis. Progress in Particle and Nuclear Physics, 107:109-166, Jul 2019.

[7] H. Schatz, A. Aprahamian, J. Goerres, M. Wiescher, T. Rauscher, J. F. Rembges, F. K. Thielemann, B. Pfeiffer, P. Moeller, K. L. Kratz, H. Herndl, B. A. Brown, and H. Rebel. rp-Process Nucleosynthesis at Extreme Temperature and Density Conditions. Physics Reports, 294:167-264, February 1998.

[8] Christian Iliadis, Art Champagne, Jordi José, Sumner Starrfield, and Paul Tupper. The Effects of Thermonuclear Reaction-Rate Variations on Nova Nucleosynthesis: A Sensitivity Study. The Astrophysical Journal Supplement Series, 142(1):105-137, September 2002.

[9] M. R. Mumpower, R. Surman, G. C. McLaughlin, and A. Aprahamian. The impact of individual nuclear properties on r-process nucleosynthesis. Progress in Particle and Nuclear Physics, 86:86-126, January 2016.

[10] R. Surman, M. Mumpower, and A. Aprahamian. Uncorrelated Nuclear Mass Uncertainties and r-process Abundance Predictions. Acta Physica Polonica B, 47(3):673, January 2016.

[11] D. Martin, A. Arcones, W. Nazarewicz, and E. Olsen. Impact of Nuclear Mass Uncertainties on the $\mathrm{r}$ Process. Physical Review Letters, 116(12):121101, March 2016.

[12] J. Bliss, A. Arcones, F. Montes, and J. Pereira. Impact of $(\alpha, \mathrm{n})$ reactions on weak r-process in neutrinodriven winds. Journal of Physics G Nuclear Physics, 44(5):054003, May 2017.
[13] Pavel Denissenkov, Georgios Perdikakis, Falk Herwig, Hendrik Schatz, Christian Ritter, Marco Pignatari, Samuel Jones, Stylianos Nikas, and Artemis Spyrou. The impact of $(n, \gamma)$ reaction rate uncertainties of unstable isotopes near $\mathrm{N}=50$ on the i-process nucleosynthesis in He-shell flash white dwarfs. Journal of Physics $G$ Nuclear Physics, 45(5):055203, May 2018.

[14] T. M. Sprouse, R. Navarro Perez, R. Surman, M. R. Mumpower, G. C. McLaughlin, and N. Schunck. Propagation of Statistical Uncertainties of Skyrme Mass Models to Simulations of $r$-Process Nucleosynthesis. arXiv e-prints, page arXiv:1901.10337, Jan 2019.

[15] Shinya Wanajo. Cold r-Process in Neutrino-driven Winds. The Astrophysical Journal Letters, 666(2):L77L80, Sep 2007.

[16] M. R. Mumpower, T. Kawano, T. M. Sprouse, N. Vassh, E. M. Holmbeck, R. Surman, and P. Möller. $\beta$-delayed Fission in r-process Nucleosynthesis. The Astrophysical Journal, 869(1):14, Dec 2018.

[17] N. Vassh, R. Vogt, R. Surman, J. Randrup, T. M. Sprouse, M. R. Mumpower, P. Jaffke, D. Shaw, E. M. Holmbeck, Y. Zhu, and G. C. McLaughlin. Using excitation-energy dependent fission yields to identify key fissioning nuclei in r-process nucleosynthesis. Journal of Physics G Nuclear Physics, 46(6):065202, Jun 2019.

[18] W. R. Hix and F. K. Thielemann. Computational methods for nucleosynthesis and nuclear energy generation. Journal of Computational and Applied Mathematics, 109(1):321-351, Sep 1999.

[19] W. D. Arnett and J. W. Truran. Carbon-Burning Nucleosynthesis at Constant Temperature. The Astrophysical Journal, 157:339, Jul 1969.

[20] S. E. Woosley, W. David Arnett, and Donald D. Clayton. The Explosive Burning of Oxygen and Silicon. The Astrophysical Journal Supplement Series, 26:231, Nov 1973.

[21] W. Raphael Hix and Bradley S. Meyer. Thermonuclear kinetics in astrophysics. Nuclear Physics A, 777:188-207, Oct 2006.

[22] Jonas Lippuner and Luke F. Roberts. SkyNet: A Modular Nuclear Reaction Network Library. The Astrophysical Journal Supplement Series, 233(2):18, Dec 2017.

[23] F. X. Timmes. Integration of Nuclear Reaction Networks for Stellar Hydrodynamics. The Astrophysical Journal Supplement Series, 124(1):241-263, Sep 1999.

[24] Mike Guidry. Algebraic stabilization of explicit numerical integration for extremely stiff reaction networks. Journal of Computational Physics, 231(16):5266-5288, June 2012.

[25] M. W. Guidry, R. Budiardja, E. Feger, J. J. Billings, W. R. Hix, O. E. B. Messer, K. J. Roche, E. McMahon, and M. He. Explicit integration of extremely stiff reaction networks: asymptotic methods. Computational Science and Discovery, 6(1):015001, January 2013.

[26] M. W. Guidry and J. A. Harris. Explicit integration of extremely stiff reaction networks: quasi-steady-state methods. Computational Science and Discovery, 6(1):015002, January 2013.

[27] M. W. Guidry, J. J. Billings, and W. R. Hix. Explicit integration of extremely stiff reaction networks: partial equilibrium methods. Computational Science and Discovery, 6(1):015003, January 2013. 
[28] Benjamin Brock, Andrew Belt, Jay Jay Billings, and Mike Guidry. Explicit integration with GPU acceleration for large kinetic networks. Journal of Computational Physics, 302:591-602, December 2015.

[29] M. R. Mumpower, T. Kawano, J. L. Ullmann, M. Krtička, and T. M. Sprouse. Estimation of M 1 scissors mode strength for deformed nuclei in the medium- to heavy-mass region by statistical Hauser-Feshbach model calculations. Physical Review C, 96(2):024612, Aug 2017.

[30] Y. Zhu, R. T. Wollaeger, N. Vassh, R. Surman, T. M. Sprouse, M. R. Mumpower, P. Möller, G. C. McLaughlin, O. Korobkin, T. Kawano, P. J. Jaffke, E. M. Holmbeck, C. L. Fryer, W. P. Even, A. J. Couture, and J. Barnes. Californium-254 and Kilonova Light Curves. Astrophysical Journal Letters, 863(2):L23, August 2018.

[31] Richard H. Cyburt, A. Matthew Amthor, Ryan Ferguson, Zach Meisel, Karl Smith, Scott Warren, Alexand er Heger, R. D. Hoffman, Thomas Rauscher, Alexand er Sakharuk, Hendrik Schatz, F. K. Thielemann, and Michael Wiescher. The JINA REACLIB Database: Its Recent Updates and Impact on Type-I X-ray Bursts. The Astrophysical Journal Supplement, 189(1):240-252, July 2010.

[32] M. R. Mumpower, T. Kawano, and P. Möller. Neutron- $\gamma$ competition for $\beta$-delayed neutron emission. Phys. Rev. C, 94:064317, Dec 2016.

[33] P. Möller, M. R. Mumpower, T. Kawano, and W. D. Myers. Nuclear properties for astrophysical and radioactiveion-beam applications (ii). At. Data Nucl. Data Tables, 125:1-192, 2019.

[34] Meng Wang, G. Audi, F. G. Kondev, W. J. Huang, S. Naimi, and Xing Xu. The AME2016 atomic mass evaluation (II). Tables, graphs and references. Chinese Phys. C, 41(3):030003, 2017.

[35] P. Möller, W. D. Myers, H. Sagawa, and S. Yoshida. Physical Review Letters, 108(5):052501, February 2012.

[36] T. Kawano, R. Capote, S. Hilaire, and P. Chau Huu-Tai. Physical Review C, 94(1):014612, July 2016.

[37] M. R. Mumpower, P. Jaffke, M. Verriere, and J. Randrup. Primary fission fragment mass yields across the chart of nuclides. Physical Review C, 101:054607, May 2020.

[38] G. Audi et al. The nubase2016 evaluation of nuclear properties. Chinese Physics C, 41(3):030001, March 2017.

[39] N.R. Council, D.E.P. Sciences, B.P. Astronomy, and C.P. Universe. Connecting Quarks with the Cosmos: Eleven Science Questions for the New Century. National Academies Press, 2003.

[40] T.C.A.O.N. Physics, B.P. Astronomy, D.E.P. Sciences, and N.R. Council. Nuclear Physics: Exploring the Heart of Matter. National Academies Press, 2013.

[41] B. P. Abbott et al. Gw170817: Observation of gravitational waves from a binary neutron star inspiral. Phys. Rev. Lett., 119:161101, Oct 2017.

[42] P. S. Cowperthwaite et al. The electromagnetic counterpart of the binary neutron star merger ligo/virgo gw170817. ii. uv, optical, and near-infrared light curves and comparison to kilonova models. The Astrophysical Journal Letters, 848(2):L17, 2017.

[43] J. Beun, G. C. McLaughlin, R. Surman, and W. R. Hix. Fission cycling in a supernova r process. Physical Review C, 77(3):035804, March 2008.

[44] O. Korobkin, S. Rosswog, A. Arcones, and C. Winteler. On the astrophysical robustness of the neutron star merger r-process. Monthly Notices of the Royal As- tronomical Society, 426(3):1940-1949, November 2012.

[45] S. Goriely. The fundamental role of fission during rprocess nucleosynthesis in neutron star mergers. European Physical Journal A, 51:22, February 2015.

[46] M. Eichler, A. Arcones, A. Kelic, O. Korobkin, K. Langanke, T. Marketin, G. Martinez-Pinedo, I. Panov, T. Rauscher, S. Rosswog, C. Winteler, N. T. Zinner, and F. K. Thielemann. The Role of Fission in Neutron Star Mergers and Its Impact on the r-Process Peaks. Astrophysical Journal, 808(1):30, July 2015.

[47] Nicole Vassh, Matthew R. Mumpower, Gail C. McLaughlin, Trevor M. Sprouse, and Rebecca Surman. Coproduction of light and heavy $r$-process elements via fission deposition. arXiv e-prints, page arXiv:1911.07766, November 2019.

[48] Karl-Heinz Schmidt and Beatriz Jurado. Review on the progress in nuclear fission - experimental methods and theoretical descriptions. Reports on Progress in Physics, 81(10):106301, October 2018.

[49] Samuel A. Giuliani, Gabriel Martínez-Pinedo, and Luis M. Robledo. Fission properties of superheavy nuclei for $r$-process calculations. Physical Review C, 97:034323, Mar 2018.

[50] J. F. Lemaître, S. Goriely, S. Hilaire, and N. Dubray. Microscopic description of the fission path with the Gogny interaction. Physical Review C, 98(2):024623, August 2018.

[51] J. F. Lemaître, S. Goriely, S. Hilaire, and J. L. Sida. Fully microscopic scission-point model to predict fission fragment observables. Physical Review C, 99(3):034612, March 2019.

[52] Jhilam Sadhukhan, Samuel A. Giuliani, Zachary Matheson, and Witold Nazarewicz. Efficient method for estimation of fission fragment yields of r-process nuclei. arXiv e-prints, page arXiv:2001.08616, January 2020.

[53] Tsvi Piran, Ehud Nakar, and Stephan Rosswog. The electromagnetic signals of compact binary mergers. Monthly Notices of the Royal Astronomical Society, 430(3):21212136, Apr 2013.

[54] Jochen Erler, Noah Birge, Markus Kortelainen, Witold Nazarewicz, Erik Olsen, Alexander M. Perhac, and Mario Stoitsov. The limits of the nuclear landscape. Nature, 486(7404):509-512, June 2012.

[55] J. D. McDonnell, N. Schunck, D. Higdon, J. Sarich, S. M. Wild, and W. Nazarewicz. Uncertainty Quantification for Nuclear Density Functional Theory and Information Content of New Measurements. Physical Review Letters, 114(12):122501, March 2015.

[56] Léo Neufcourt, Yuchen Cao, Samuel A. Giuliani, Witold Nazarewicz, Erik Olsen, and Oleg B. Tarasov. Quantified limits of the nuclear landscape. arXiv e-prints, page arXiv:2001.05924, January 2020.

[57] N. D. Scielzo, G. Li, M. G. Sternberg, G. Savard, P. F. Bertone, F. Buchinger, S. Caldwell, J. A. Clark, J. Crawford, C. M. Deibel, J. Fallis, J. P. Greene, S. Gulick, A. A. Hecht, D. Lascar, J. K. P. Lee, A. F. Levand, M. Pedretti, R. E. Segel, H. Sharma, K. S. Sharma, I. Tanihata, J. Van Schelt, R. M. Yee, and B. J. Zabransky. The $\beta$-decay Paul trap: A radiofrequency-quadrupole ion trap for precision $\beta$-decay studies. Nuclear Instruments and Methods in Physics Research A, 681:94-100, July 2012.

[58] R. M. Yee, N. D. Scielzo, P. F. Bertone, F. Buchinger, S. Caldwell, J. A. Clark, C. M. Deibel, J. Fallis, J. P. Greene, S. Gulick, D. Lascar, A. F. Levand, G. Li, 
E. B. Norman, M. Pedretti, G. Savard, R. E. Segel, K. S. Sharma, M. G. Sternberg, J. Van Schelt, and B. J. Zabransky. $\beta$-Delayed Neutron Spectroscopy Using Trapped Radioactive Ions. Physical Review Letters, 110(9):092501, March 2013.

[59] N. D. Scielzo, R. M. Yee, P. F. Bertone, F. Buchinger, S. A. Caldwell, J. A. Clark, A. Czeszumska, C. M. Deibel, J. P. Greene, S. Gulick, D. Lascar, A. F. Levand, G. Li, E. B. Norman, S. Padgett, M. Pedretti, A. Perez Galvan, G. Savard, R. E. Segel, K. S. Sharma, M. G. Sternberg, J. Van Schelt, and B. J. Zabransky. A Novel Approach to $\beta$-delayed Neutron Spectroscopy Using the Beta-decay Paul Trap. Nuclear Data Sheets, 120:70-73, June 2014.

[60] B. S. Wang, S. A. Caldwell, N. D. Scielzo, A. Czeszumska, J. A. Clark, G. Savard, A. Aprahamian, M. T. Burkey, C. J. Chiara, J. Harker, A. F. Levand, S. T. Marley, G. E. Morgan, J. M. Munson, E. B. Norman, A. Nystrom, R. Orford, S. W. Padgett, A. Pérez Galván, K. S. Sharma, K. Siegl, and S. Y. Strauss. $\beta$-delayed-neutron studies of ${ }^{S b}, 136_{135}$ and ${ }^{140}$ I performed with trapped ions. Physical Review C, 101(2):025806, February 2020.

[61] T. Y. Hirsh, N. Paul, M. Burkey, A. Aprahamian, F. Buchinger, S. Caldwell, J. A. Clark, A. F. Levand, L. L. Ying, S. T. Marley, G. E. Morgan, A. Nystrom, R. Orford, A. P. Galván, J. Rohrer, G. Savard, K. S. Sharma, and K. Siegl. First operation and mass separation with the CARIBU MR-TOF. Nuclear Instruments and Methods in Physics Research B, 376:229-232, June 2016.

[62] R. Orford, N. Vassh, J. A. Clark, G. C. McLaughlin, M. R. Mumpower, G. Savard, R. Surman, A. Aprahamian, F. Buchinger, M. T. Burkey, D. A. Gorelov, T. Y. Hirsh, J. W. Klimes, G. E. Morgan, A. Nystrom, and K. S. Sharma. Precision Mass Measurements of Neutron-Rich Neodymium and Samarium Isotopes and Their Role in Understanding Rare-Earth Peak Formation. Physical Review Letters, 120(26):262702, June 2018.

[63] G. Savard, M. Brodeur, J. A. Clark, R. A. Knaack, and A. A. Valverde. The $\mathrm{N}=126$ factory: A new facility to produce very heavy neutron-rich isotopes. Nuclear Instruments and Methods in Physics Research B, 463:258261, January 2020.

[64] A. Kankainen, J. Hakala, T. Eronen, D. Gorelov, A. Jokinen, V. S. Kolhinen, I. D. Moore, H. Penttilä, S. RintaAntila, J. Rissanen, A. Saastamoinen, V. Sonnenschein, and J. Äystö. Isomeric states close to doubly magic ${ }^{132} \mathrm{Sn}$ studied with the double Penning trap JYFLTRAP. Phys. Rev. C, 87(2):024307, February 2013.

[65] M. Vilen, J. M. Kelly, A. Kankainen, M. Brodeur, A. Aprahamian, L. Canete, T. Eronen, A. Jokinen, T. Kuta, I. D. Moore, M. R. Mumpower, D. A. Nesterenko, H. Penttilä, I. Pohjalainen, W. S. Porter, S. Rinta-Antila, R. Surman, A. Voss, and J. ńystö. Precision Mass Measurements on Neutron-Rich Rare-Earth Isotopes at JYFLTRAP: Reduced Neutron Pairing and Implications for $\mathrm{r}$-Process Calculations. Physical Review Letters, 120(26):262701, June 2018.

[66] D. Lunney and (on behalf of ISOLTRAP Collaboration. Extending and refining the nuclear mass surface with ISOLTRAP. Journal of Physics G Nuclear Physics, 44(6):064008, June 2017.

[67] D. Lascar, R. Klawitter, C. Babcock, E. Leistenschneider, S. R. Stroberg, B. R. Barquest, A. Finlay, M. Foster, A. T. Gallant, P. Hunt, J. Kelly, B. Kootte, Y. Lan,
S. F. Paul, M. L. Phan, M. P. Reiter, B. Schultz, D. Short, J. Simonis, C. Andreoiu, M. Brodeur, I. Dillmann, G. Gwinner, J. D. Holt, A. A. Kwiatkowski, K. G. Leach, and J. Dilling. Precision mass measurements of ${ }^{125-127} \mathrm{Cd}$ isotopes and isomers approaching the $N=82$ closed shell. ArXiv e-prints, May 2017.

[68] B. Moon, C. B. Moon, P. A. Söderström, A. Odahara, R. Lozeva, B. Hong, F. Browne, H. S. Jung, P. Lee, C. S. Lee, A. Yagi, C. Yuan, S. Nishimura, P. Doornenbal, G. Lorusso, T. Sumikama, H. Watanabe, I. Kojouharov, T. Isobe, H. Baba, H. Sakurai, R. Daido, Y. Fang, H. Nishibata, Z. Patel, S. Rice, L. Sinclair, J. Wu, Z. Y. Xu, R. Yokoyama, T. Kubo, N. Inabe, H. Suzuki, N. Fukuda, D. Kameda, H. Takeda, D. S. Ahn, Y. Shimizu, D. Murai, F. L. Bello Garrote, J. M. Daugas, F. Didierjean, E. Ideguchi, T. Ishigaki, S. Morimoto, M. Niikura, I. Nishizuka, T. Komatsubara, Y. K. Kwon, and K. Tshoo. Nuclear structure and $\beta$-decay schemes for Te nuclides beyond $\mathrm{N}=82$. Physical Review C, 95(4):044322, April 2017.

[69] Naoki Fukuda, Toshiyuki Kubo, Daisuke Kameda, Naohito Inabe, Hiroshi Suzuki, Yohei Shimizu, Hiroyuki Takeda, Kensuke Kusaka, Yoshiyuki Yanagisawa, Masao Ohtake, Kanenobu Tanaka, Koichi Yoshida, Hiromi Sato, Hidetada Baba, Meiko Kurokawa, Tetsuya Ohnishi, Naohito Iwasa, Ayuko Chiba, Taku Yamada, Eiji Ideguchi, Shintaro Go, Rin Yokoyama, Toshihiko Fujii, Hiroki Nishibata, Kazuo Ieki, Daichi Murai, Sadao Momota, Daiki Nishimura, Yoshiteru Sato, Jongwon Hwang, Sunji Kim, Oleg B. Tarasov, David J. Morrissey, and Gary Simpson. Identification of New Neutron-Rich Isotopes in the Rare-Earth Region Produced by 345 $\mathrm{MeV} /$ nucleon ${ }^{238} \mathrm{U}$. Journal of the Physical Society of Japan, 87(1):014202, January 2018.

[70] A. Tolosa-Delgado, J. Agramunt, J. L. Tain, A. Algora, C. Domingo-Pardo, A. I. Morales, B. Rubio, A. Tarifeño-Saldivia, F. Calviño, G. Cortes, N. T. Brewer, B. C. Rasco, K. P. Rykaczewski, D. W. Stracener, J. M. Allmond, R. Grzywacz, R. Yokoyama, M. Singh, T. King, M. Madurga, S. Nishimura, V. H. Phong, S. Go, J. Liu, K. Matsui, H. Sakurai, G. G. Kiss, T. Isobe, H. Baba, S. Kubono, N. Fukuda, D. S. Ahn, Y. Shimizu, T. Sumikama, H. Suzuki, H. Takeda, P. A. Söderström, M. Takechi, C. G. Bruno, T. Davinson, C. J. Griffin, O. Hall, D. Kahl, P. J. Woods, P. J. Coleman-Smith, M. Labiche, I. Lazarus, P. Morrall, V. F. E. Pucknell, J. Simpson, S. L. Thomas, M. Prydderch, L. J. HarknessBrennan, R. D. Page, I. Dillmann, R. Caballero-Folch, Y. Saito, A. Estrade, N. Nepal, F. Montes, G. Lorusso, J. Liang, S. Bae, J. Ha, B. Moon, and Briken Collaboration. Commissioning of the BRIKEN detector for the measurement of very exotic $\beta$-delayed neutron emitters. Nuclear Instruments and Methods in Physics Research A, 925:133-147, May 2019.

[71] Horst Stöcker, Thomas Stöhlker, and Christian Sturm. FAIR - Cosmic Matter in the Laboratory. In Journal of Physics Conference Series, volume 623 of Journal of Physics Conference Series, page 012026, June 2015.

[72] R. Caballero-Folch, C. Domingo-Pardo, J. Agramunt, A. Algora, F. Ameil, Y. Ayyad, J. Benlliure, M. Bowry, F. Calviño, D. Cano-Ott, G. Cortès, T. Davinson, I. Dillmann, A. Estrade, A. Evdokimov, T. Faestermann, F. Farinon, D. Galaviz, A. R. García, H. Geissel, W. Gelletly, R. Gernhäuser, M. B. Gómez-Hornillos, C. Guer- 
rero, M. Heil, C. Hinke, R. Knöbel, I. Kojouharov, J. Kurcewicz, N. Kurz, Yu. A. Litvinov, L. Maier, J. Marganiec, M. Marta, T. Martínez, F. Montes, I. Mukha, D. R. Napoli, C. Nociforo, C. Paradela, S. Pietri, Zs. Podolyák, A. Prochazka, S. Rice, A. Riego, B. Rubio, H. Schaffner, Ch. Scheidenberger, K. Smith, E. Sokol, K. Steiger, B. Sun, J. L. Taín, M. Takechi, D. Testov, H. Weick, E. Wilson, J. S. Winfield, R. Wood, P. J. Woods, and A. Yeremin. $\beta$-decay half-lives and $\beta$ delayed neutron emission probabilities for several isotopes of $\mathrm{Au}, \mathrm{Hg}, \mathrm{Tl}, \mathrm{Pb}$, and $\mathrm{Bi}$, beyond $\mathrm{N}=126$. Physical Review C, 95(6):064322, June 2017.

[73] A. Gottardo, J. J. Valiente-Dobón, G. Benzoni, A. I. Morales, A. Gadea, S. Lunardi, P. Boutachkov, A. M. Bruce, M. Górska, J. Grebosz, S. Pietri, Zs. Podolyák, M. Pfützner, P. H. Regan, D. Rudolph, H. Weick, J. Alcántara Núñez, A. Algora, N. Al-Dahan, G. de Angelis, Y. Ayyad, N. Alkhomashi, P. R. P. Allegro, D. Bazzacco, J. Benlliure, M. Bowry, A. Bracco, M. Bunce, F. Camera, E. Casarejos, M. L. Cortes, F. C. L. Crespi, A. Corsi, A. M. Denis Bacelar, A. Y. Deo, C. Domingo-Pardo, M. Doncel, Zs. Dombradi, T. Engert, K. Eppinger, G. F. Farrelly, F. Farinon, H. Geissel, J. Gerl, N. Goel, E. Gregor, T. Habermann, R. Hoischen, R. Janik, S. Klupp, I. Kojouharov, N. Kurz, S. M. Lenzi, S. Leoni, S. Mandal, R. Menegazzo, D. Mengoni, B. Million, D. R. Napoli, F. Naqvi, C. Nociforo, A. Proc- hazka, W. Prokopowicz, F. Recchia, R. V. Ribas, M. W. Reed, E. Sahin, H. Schaffner, A. Sharma, B. Sitar, D. Siwal, K. Steiger, P. Strmen, T. P. D. Swan, I. Szarka, C. A. Ur, P. M. Walker, O. Wieland, H. J. Wollersheim, F. Nowacki, and E. Maglione. New spectroscopic information on ${ }^{T l}, 213_{211}$ : A changing structure beyond the $\mathrm{N}=126$ shell closure. Physical Review C, 99(5):054326, May 2019.

[74] https://groups.nscl.msu.edu/frib/rates/fribrates.html.

[75] Rebecca Surman, Jonathan Engel, Jonathan R. Bennett, and Bradley S. Meyer. Source of the rare-earth element peak in $r$-process nucleosynthesis. Phys. Rev. Lett., 79:1809-1812, Sep 1997.

[76] R. Surman and J. Engel. Changes in r-process abundances at late times. Phys. Rev. C, 64:035801, Aug 2001.

[77] A. Arcones and G. Martínez-Pinedo. Dynamical $r$ process studies within the neutrino-driven wind scenario and its sensitivity to the nuclear physics input. Physical Review C, 83:045809, Apr 2011.

[78] Matthew R. Mumpower, G. C. McLaughlin, and Rebecca Surman. Formation of the rare-earth peak: Gaining insight into late-time $r$-process dynamics. Physical Review C, 85:045801, Apr 2012.

[79] M. Mumpower, J. Cass, G. Passucci, R. Surman, and A. Aprahamian. Sensitivity studies for the main $\mathrm{r}$ process: $\beta$-decay rates. AIP Advances, 4(4):041009, 2014. 